\title{
Seiberg-Witten Integrable Systems
}

\author{
Ron Y. Donagi* ${ }^{*}$ \\ Department of Mathemtics \\ University of Pennsylvania \\ Philadelphia, PA 19104-6395
}

\section{Introduction}

While many mathematicians are aware of the monopole equations [W] and of the impact they have had in topology, probably not very many are as familiar with the works [SW1, SW2] of Seiberg and Witten on which the monopole equations are based. The appearance of these works was a major breakthrough in Quantum Field Theory (QFT), and has to a large extent revolutionized and transformed the subject. The actual achievement in [SW1] was an exact solution to a problem in QFT (pure $N=2$ supersymmetric Yang-Mills in four dimensions with gauge group $\mathrm{SU}(2)$ ) which could traditionally be solved only approximately, by computing the first few terms in a perturbative expansion. The solution is close to algebraic geometry both in its details (involving families of elliptic curves) and in its general approach, which is to use global properties (monodromy, symmetries) of a physical theory to uniquely characterize a geometric model of the theory. This work opened the doors to solutions, along similar lines, of many specific quantum theories, some of which are reviewed here. It also led directly to the ongoing revolution in string theory involving string dualities and the appearance of higher dimensional objects (branes) on more or less equal footing with the strings themselves.

${ }^{*}$ Work partially supported by NSF grant DMS95-03249, and by grants from the Univesity of Pennsylvania Research Foundation and The Harmon Duncombe Foundation.

†Current address: School of Mathematics, Institute for Advanced Study, Princeton, NJ 08540 
The purpose of these notes is to try to make this exciting and rapidly evolving chapter of high energy physics accessible to mathematicians, particularly to algebraic geometers. I have had to make some choices: I decided to focus on the original work and its further development within QFT, thus omitting the spectacular (but well documented) applications to topology and strings. Even so, it was impossible to render with mathematical rigor the enormous amount of background material needed from the physics. I opted instead, in $\S 1$, for the telling of one uniform but very imprecise story of the physics. My hope is that it will afford, to a mathematical reader with no prior knowledge of the physics, at least a general sense of the physical theory, of how the different parts interact, and of what some of the basic concepts mean. The remaining, mathematical, sections of these notes discuss what is now known (and what is still missing) about Seiberg-Witten type solutions of four-dimensional $N=2$ supersymmetric Yang-Mills theories in algebro-geometric terms, with the emphasis on an interpretation via integrable systems.

Section 1 starts with Maxwell's classical description of electromagnetism. We arrive at our central object of study, the duality in super Yang-Mills theory, after discussing separately the three directions in which Maxwell's theory needs to be extended: nonabelian gauge groups, quantization, and supersymmetry. We are then ready for an overview of the Seiberg-Witten solution, [SW1], emphasizing its algebro-geometric elements. The section concludes with a discussion of the other theories which could be exactly solvable by algebro-geometric techniques. These theories occupy us in the remainder of these notes.

Algebraically integrable systems are quickly reviewed in $\S 2$. The upshot is that all the structures arising in the Seiberg-Witten physics of $\S 1$ are naturally realized here, so algebraically integrable systems are the natural context for describing the physics (cf. [DW], $[\mathrm{F}]$ ). We also clarify the role of the SeibergWitten differential, which occurs naturally in any integrable system of spectral type. One important feature of this differential, the linear dependence of its residues on the mass parameters, turns out to be part of a very general phenomenon. We state and outline a proof of an apparently new result, which is a complex analogue of the theorem of Duistermaat-Heckman $[\mathrm{DH}]$ on variation of the symplectic structure in a family of symplectic quotients. The complex case is somewhat subtler than the compact version due to the presence of nonsemisimple (e.g. nilpotent) orbits.

Section 3 is mostly an attempt to rewrite the solution [DW] of the $\mathrm{SU}(n)$ theory with adjoint matter, with an emphasis on the math rather than the physics. We review the work of Markman [Mn] and others on the integrable systems of meromorphic Higgs bundles; among these systems we pick the particular subsystem we need. The system (i.e. its spectral curves) is described explicitly, then compared to the elliptic soliton description [T, TV]. A major part of [DW] conisted of "tests" showing that this system accurately reproduces physical properties of the theory; we describe some of these "tests" here.

In the final section we discuss the other major class of integrable systems which is known to solve the pure $N=2$ SYM theories: the periodic Toda 
lattices [MW1]. In particular, we compare the various forms assumed by the spectral curves. We then move to a common generalization of both systems, the elliptic Calogero-Moser systems, which were proposed in [Mc] as a solution of the theory with adjoint matter and arbitrary gauge group. This seems quite promising, but it still lacks a satisfactory algebro-geometric description. We also mention briefly some other theories where at least the family of spectral curves is known, and conclude by mentioning some recent ideas in string theory which exhibit the QFT integrable systems discussed here as limits of stringy systems.

It is a pleasure to acknowledge pleasant and useful conversations, about some of the topics covered here, with D. Freed, L. Jeffrey, A. Kirillov, D. Lowe, N. Nekrasov, R. Plesser, N. Seiberg, S. Singer, R. Sjammar, J. Weitsman, and E. Witten, as well as helpful comments on the manuscript by D. Freed, A. Grassi, A. Ksir, N. Nekrasov, M. Verbitsky and E. Witten.

\section{Some Physics Background}

\subsection{Electromagnetism}

The electric field $E$ and the magnetic field $B$ in $\mathbb{R}^{3}$ are governed by Maxwell's equations, of T-shirt fame. Think of $E$ and $B$, respectively, as a 1-form and a 2 -form on $\mathbb{R}^{3}$. Both are time-dependent, so we combine them into the electromagnetic field $F:=E \wedge d t+B$, a 2 -form on $\mathbb{R}^{4}$. Maxwell's equations in vacuo are:

$$
d F=0 \quad d * F=0
$$

Here $*$ is the Hodge star operator on forms on $\mathbb{R}^{4}$, determined by the Minkowski metric $g=d x^{2}+d y^{2}+d z^{2}-c^{2} d t^{2}$. (The speed of light $c$ is usually set to 1 by adjusting units.)

The same equations make sense for an unknown 2-form $F$ on any (pseudo) Riemannian manifold $(X, g)$. They are clearly invariant under the group of global isometries of $(X, g)$. When $\operatorname{dim} X=4$, the equations are also invariant under the duality $F \mapsto * F$. When $X=\mathbb{R}^{4}$ with the Minkowski metric, this duality interchanges the electric and magnetic fields. The group of isometries in this case is the Poincare group, an extension of the Lorentz group $\mathrm{SO}(3,1)$ of rotations by the group $\left(\mathbb{R}^{4},+\right)$ of translations.

Geometrically, we fix a $\mathrm{U}(1)$-bundle $L$ on $X$ and consider a connection $A$ on $L$ as a new variable. The first equation $d F=0$ is interpreted as saying that (locally) $F=d A$ is the curvature. The remaining equation then is $d * d A=0$. The group of isometries of the triple $(X, g, L)$ acts on the connections and preserves the equation. This is an extension of the group of those isometries of $(X, g)$ which preserve $L$ by the gauge group of $C^{\infty}$ maps from $X$ to $\mathrm{U}(1)$. The duality is still there, but is now hidden.

Now impose an external field $j$. (In $\mathbb{R}^{4}, j$ is a 3 -form whose $d x \wedge d y \wedge d z$ component is the charge density, while the other 3 components give the current.) 
The equations become

$$
d F=0, \quad d * F=j
$$

and duality is lost. Geometrically, once we think of $F$ as curvature, the first equation becomes a tautology (the Bianchi identity), while the second is an extra condition which may or may not hold. Physically, we explain this asymmetry as due to the absence of magnetic charges ("monopoles").

Dirac proposed that some magnetic charges can be introduced by changing the topology, or allowing singularities. For example, a monopole at the origin of $\mathbb{R}^{3}$ is represented by a $\mathrm{U}(1)$-bundle on $\left(\mathbb{R}_{x, y, z}^{3} \backslash 0\right) \times \mathbb{R}_{t}$. Such bundles are characterized topologically by their Chern class $c_{1} \in \mathbb{Z}$, and this $c_{1}$ is interpreted as the magnetic charge at the origin. Indeed, the "magnetic charge" at the origin is just the total magnetic flux across a small sphere $S^{2}$ around the origin in $\mathbb{R}^{3}$; this is the integral over $S^{2}$ of $B$, which equals the integral of $F$, which is $c_{1}$. We can extend $F$ to $\mathbb{R}^{4}$ as a distribution, but now $d F$ is the $\delta$-function $c_{1} . \delta_{x, y, z}$ instead of $d F=0$. This is not yet sufficient to restore the symmetry to Maxwell's equations: the electric charge is arbitrary, but the magnetic charge is allowed only in discrete "quanta". This does raise the possibility, though, that complete symmetry could be restored in a quantum theory, where, as in the real world, electric charge also comes in discrete units. Indeed, Dirac discovered that in quantum mechanics, where an electrically charged particle is represented by a wave function satisfying Schrödinger's equation, the electric charge $e$ of one particle and the magnetic charge $g$ of another (in appropriate units) satisfy $e g \in \mathbb{Z}$. This has the curious interpretation that all electric charges are automatically quantized as soon as one magnetic monopole exists somewhere in the universe.

In [MO], Montonen and Olive conjectured that in certain supersymmetric Yang-Mills quantum field theories the electric-magnetic duality is indeed restored. This involves an extension of the basic picture in three separate directions: replacing the "abelian" Maxwell equations by the non-abelian Yang-Mills; quantizing the classical theory; and adding supersymmetry to the Poincare invariance.

\section{$1.2 \quad$ Yang-Mills theory}

Yang-Mills theory is quite familiar to mathematicians. It involves replacing the structure group $U(1)$ by an arbitrary reductive group $G$. We thus have a connection $A$ on some $G$-bundle $V$ over our fourfold $X$, the curvature is now the $\operatorname{ad}(V)$-valued two-form $d A+\frac{1}{2}[A, A]$, and the equations are as before, $d F=0$, $d * F=0$, except that the covariant derivative $d=d_{A}$ on $\operatorname{ad}(V)$ now replaces the exterior derivative $d$ on the trivial bundle $\operatorname{ad}(L)$.

These equations can be, and usually are, considered as variational equations for the Lagrangian

$$
L(A):=\int_{X} \operatorname{Tr}(F \wedge * F)
$$


where the trace $\operatorname{Tr}$ can be any non-degenerate invariant pairing on the Lie algebra $\mathfrak{g}$ of $G$. This Lagrangian is considered as a functional on an appropriate space of connections $A$ (if $X$ is not compact, we must impose some decay condition at infinity for the integral to converge). The equation $d F=0$ is again the Bianchi identity, while $d * F=0$ is the equation of the critical locus of $L$. Either from the Lagrangian description or directly from the equations, it is clear that the symmetry group includes global isometries of $X$ which lift to $V$ ("the Poincare group") as well as local diffeomorphisms of the bundle ("the gauge group") and, in dimension 4, the duality $F \mapsto * F$. In the original model of Yang and Mills, the group $G$ was $\mathrm{SU}(2)$, while in QCD it is $\mathrm{SU}(3)$. For any "realistic" model of all of particle physics, $G$ would need to contain at least $\mathrm{U}(1) \times \mathrm{SU}(2) \times \mathrm{SU}(3)$.

\subsection{Quantization}

Quantization is an art form which, when applied to classical physical theories, yields predictions of subatomic behavior which are in spectacular agreement with experiments. The quantization of the classical mechanics of particles leads to quantum mechanics, which can be described, in a Hamiltonian approach, via operators and rays in Hilbert space, or in a Lagrangian formulation, via path integrals. Quantization of continuous fields leads to QFT: quantum field theory. It has a Hamiltonian formulation, where the Hilbert space is replaced by the "larger" Fock space, as well as a Lagrangian formulation, based on averaging the action (= exp of the Lagrangian) over "all possible histories" via path integrals. These are generally ill-defined integrals over infinite-dimensional spaces. Nevertheless, there exist powerful tools for expanding them perturbatively, as power series in some "small" parameters such as Planck's constant, around a point corresponding to a "free" limit where the integral becomes Gaussian and can be assigned a value. (Actually, in modern QFT the "small parameters" used are often taken to be dimensionless coupling constants rather than Planck's constant.) The coefficients of the expansion are finite dimensional integrals encoded combinatorially as Feynman diagrams. These power series typically diverge and need to be "regularized" and "renormalized". These processes are somewhat akin to the way we make the Weierstrass $\mathfrak{P}$-function converge, by subtracting a correction term from each summand in an infinite series: a first attempt to write down a doubly periodic meromorphic function in terms of an infinite series of functions leads, unfortunately, to an everywhere divergent series; nevertheless we can obtain a series which does converge, and indeed to a doubly periodic meromorphic function, by subtracting an appropriate constant from each term. A physicist might say that we "cancelled the infinity" of the original series by subtracting "an infinite constant". In any case, it is some of these renormalized values which are confirmed to amazing accuracy by experiment, providing physicists with unshakable confidence in the validity of the technique.

For a physicist, a "theory", either classical or quantum, is usually specified 
by its Lagrangian $L$, a functional given by integration over the underlying space $X$ of a Lagrangian density $\mathcal{L}$, which is a local expression involving the values and derivatives of a given collection of fields. The classical theory considers extrema of $L$, while the quantum theory averages the action over all possible fields. The largest weight is thus still assigned to the extrema of $L$, but all "quantum fluctuations" around the extrema are now included. The Lagrangian density typically consists of a quadratic part, plus higher order terms which in a perturbative approach are interpreted as small perturbations. In a theory consisting of scalar fields $\varphi_{i}$, the quadratic term might be $\sum_{i}\left(\left|d \varphi_{i}\right|^{2}+m_{i}^{2} \varphi_{i}^{2}\right)$, where $m_{i}$ are the masses. A perturbation might include terms like $g_{I} \varphi^{I}$, where $I=\left(i_{1}, \ldots, i_{k}\right)$ is a multiindex, and $g_{I}$ is a (supposedly small) coupling constant giving the strength of an interaction involving the $k$ fields $\varphi_{i}, i \in I$. (Other types of theories might involve also some fields which are vectors or spinors with respect to the Poincare group.) A typical quantity to be computed is the n-point function

$$
\left\langle x_{1}, \ldots, x_{n}\right\rangle:=\int \varphi\left(x_{1}\right) \ldots \varphi\left(x_{n}\right) \exp (i L(\varphi)) D \varphi
$$

where integration is over the infinite-dimensional space of all $\varphi$ 's, with respect to a (non-existent) measure $D \varphi$, and $L(\varphi):=\int_{X} \mathcal{L}(\varphi)$.

A formal approach to this integral is to interpret it as a power series in the coupling constants. Terms of the expansion are described by Feynman diagrams. The types of edges in a diagram correspond to the types of fields in the theory. The vertex types correspond to the multiindices $I$ with non-zero $g_{I}$. A $\varphi^{4}$ term thus corresponds to a vertex where four $\varphi$ edges meet (this may represent two incoming particles which collide and scatter away), while a $\varphi \psi \bar{\psi}$ vertex can represent an electron $\psi$ annihilating a positron $\bar{\psi}$ to produce a photon $\varphi$. The $F$ eynman rules assign to each diagram a $4 \ell$ dimensional integral, where $\ell$ is the number of loops, i.e. first Betti number, of the diagram, and 4 is the dimension of space-time: the integration is over all ways of assigning a momentum (a 4-vector) to each oriented edge, subject to Kirchhoff's law of null-sum at each vertex. The integrand for each diagram is the product over all edges of a basic function (the propagator) of the momentum assigned to that edge, times a factor involving the coupling constant $g_{I}$ at each vertex of type $I$, and an overall numerical factor accounting for graph automorphisms. The n-point function, or rather its Fourier tranform, is then expanded as the sum of all possible diagram integrals with $n$ external legs.

One problem is that some of these Feynman integrals may be divergent. Of course, even if they converge individually, their sum may not. So the individual integrals need to be regularized, and the entire theory needs to be renormalized. A theory is said to be superrenormalizable if all divergences in it disappear after a finite number of its divergent diagrams are made finite by adjusting some of the parameters. In a renormalizable theory there are infinitely many divergent diagrams, but they can all be renormalized by iteratively adjusting the values of just a finite collection of parameters. There is an easily verifiable necessary 
condition for renormalizability: each field $\varphi$ carries a certain dimensionality $[\varphi]$. (This is measured in powers of the mass; time and distance are converted to mass-inverse by setting the speed of light $c$ and Planck's constant $h$ to 1.) Now $L$, which occurs as an exponent, must have no dimensionality (it is a pure number, independent of units), so each term in $\mathcal{L}$ must have dimensionality 4 . This determines the dimensionality of each $g_{I}$. The obvious necessary condition for renormalizability is that $\left[g_{I}\right] \geq 0$ for all $I$ : otherwise, the insertion of a vertex of type $I$ in an already divergent diagram will give a new diagram with divergence which is worse. Similarly, a necessary condition for superrenormalizability is that $\left[g_{I}\right]>0$ for all $I$, since otherwise insertions into one divergent diagram would yield others with equally bad divergence. These conditions serve to point out the very few candidates for renormalizable theories. Actually proving renormalizability in each case tends to be much harder.

For regularization, we introduce an auxiliary variable $\Lambda$ so that the integral can be interpreted as (the "limit" of) a function of $\Lambda$ going to infinity with $\Lambda$. For example, $\Lambda$ can be a momentum cutoff, meaning that the integral is carried out only over the ball of momenta $p$ satisfying $|p| \leq \Lambda$. (Or better, use some approximation of unity by smooth compactly supported cutoff functions.) Another popular variation is dimensional regularization: one writes down the expression for a given integral in a $d$-dimensional space, and observes that the answer is an analytic function in $d$ for sufficiently small $d$, typically acquiring a singularity at the relevant dimension $d=4$. The analogue of $\Lambda$ in this version is $\exp \left(\frac{1}{4-d}\right)$.

The modern approach to renormalization is based on the renormalization group flow. Very crudely, one might illustrate this process as follows. The integral for each diagram $D$ translates into a function $f_{D}(m, g, p, \Lambda)$ of the masses, coupling constants, external momenta and cutoff (or whatever else we used for $\Lambda$ ). If the number of divergent diagrams is finite (and $\leq$ the number of parameters in $L$ ) then the simultaneous level sets of the corresponding $f_{D}$ will be (or will contain) a family of curves on which $\Lambda$ is unbounded. So we may hope to find a flow parametrized by $\Lambda$ along these curves:

$$
m=m(\Lambda), g=g(\Lambda), p=p(\Lambda) .
$$

In a general renormalizable theory there may be infinitely many $f_{D}$, but each may be modified (by a quantity which is bounded as a function of $\Lambda$ ) so there is still a flow tangent to all of them. Instead of attempting to fix the parameters at their "bare" values and taking $\Lambda$ to infinity, renormalization is accomplished by flowing along this renormalization group flow. The effective, physically measurable value of the parameters thus varies with the scale $\Lambda$. Similar phenomena are rather common in statistical physics. For example, the electric charge of a particle in a dielectric appears to be reduced, or screened, with distance, as a result of the alignment of the dielectric's charges around it. In QFT this occurs even in vacuum, because of the quantum fluctuations.

A famous series of experiments at the Stanford Linear Accelerator showed that, when bombarded by very high energy electrons, a proton behaves as if it 
is made of three separate subparticles (quarks) which move freely within it. Yet at low energies (or equivalently, as viewed on a larger distance scale), the quarks are confined within the proton. A major success of QFT was its interpretation of these results in terms of the varying coupling constant $g$ for the strong force. The relevant theory (QCD: quantum chromodynamics) is asymptotically free, which means that $g \rightarrow 0$ as $\Lambda \rightarrow \infty$, and $g \rightarrow \infty$ as $\Lambda \rightarrow 0$. Thus at high energy scale $\Lambda$, the quarks behave as if $g=0$, i.e. as in the free theory, while at large distance $\Lambda \rightarrow 0$ the force becomes stronger and is presumably sufficient to confine the quarks. (This last point is not rigorously proved; Seiberg and Witten obtained a model for confinement in the supersymmetric analogue.)

An often computable quantity which describes the behavior of a coupling constant is its beta function, $\beta:=\Lambda \partial g / \partial \Lambda$. An asymptotically free theory corresponds to $\beta<0$ for small values of $g$. In the boundary case where $\beta$ is identically 0 , the theory is scale-invariant.

An additional complication is involved in the quantization of theories which are (that is, their Lagrangian is) gauge-invariant. The problem with gaugeinvariant theories stems from the degeneracy of their Lagrangian. In classical gauge theory, the classical solutions (extrema of the action) are not isolated, but come in entire gauge-group orbits. The equivalence of the Lagrangian and Hamiltonian descriptions breaks down: this equivalence is based on a Legendre transform, which makes sense only near isolated points. In the quantized theory, the path integral needs to be taken not over all paths, but over gaugeequivalence classes. This is seen already for the free (unperturbed) theory: the value of a Gaussian integral is given by the inverse determinant of the operator in the exponent; degeneracy (or gauge invariance) implies that this determinant vanishes, and needs to be replaced by a determinant on an appropriate transversal slice or quotient. For quantized Yang-Mills, or gauge, theories, a good reference is [FS]. The reward for handling these additional complications is a collection of theories which are renormalizable and sometimes asymptotically free: the beta-function of the basic theory (corresponding to Maxwell's in vacuo) is negative. The coupling to matter increases beta, but it remains negative when the number of quarks is small enough.

\subsection{Supersymmetry}

Supermathematics is the habit of adding the prefix "super" to ordinary, commutative objects, to denote their sign-commutative generalization. Thus, a super space is a locally ringed topological space whose structure sheaf $\mathcal{O}=\mathcal{O}_{0} \oplus \mathcal{O}_{1}$ is $\mathbb{Z}_{2}$ graded, $\mathcal{O}_{0}$ is central, and multiplication $\mathcal{O}_{1} \times \mathcal{O}_{1} \rightarrow \mathcal{O}_{0}$ is skew symmetric. Affine super space $\mathbb{R}^{m, n}$ is the topologcal space $\mathbb{R}^{m}$ with the sheaf $C^{\infty}\left(\mathbb{R}^{m}\right) \otimes \Lambda^{\cdot}\left(\mathbb{R}^{n}\right)$ and the $\mathbb{Z}_{2}$ grading coming from the $\mathbb{Z}$-grading of the exterior algebra. An $(m, n)$-dimensional super manifold is a super space which is locally isomorphic to $\mathbb{R}^{m, n}$ (The isomorphisms are, of course, isomorphisms of

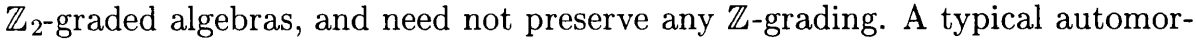
phism of $\mathbb{R}^{1,2}$, with even coordinate $x$ and odd coordinates $\theta_{1}, \theta_{2}$, may send $x$ 
to $x+\theta_{1} \theta_{2}$. So the identification of $\mathcal{O}$ as $C^{\infty}$ tensor exterior algebras is not preserved under coordinate change.) All of calculus extends to super manifolds. The only non-obvious point is that the transformation law for differential forms involves the determinant of the transformation in the even variables, but the inverse determinant of the transformation in the odd variables, and a hybridthe Berezinian - for a general transformation mixing the parities. The resulting operation of integration looks, in the odd directions, more like usual differentiation, i.e., in local coordinates $x_{1}, \ldots, x_{m}, \theta_{1}, \ldots, \theta_{n}$ it reads off the coefficient of the top odd part, $\prod_{i=1}^{n} d \theta_{i}$.

The infinitesimal symmetries of a super manifold are described by a super Lie algebra: a $\mathbb{Z}_{2}$-graded algebra $\mathfrak{g}=\mathfrak{g}_{0} \oplus \mathfrak{g}_{1}$ with a $[$,$] operation which is sign-$ antisymmetric and satisfies the only reasonable version of the Jacobi identity:

$$
\sum_{i=1}^{3}(-1)^{x_{i-1} x_{i+1}}\left[X_{i-1},\left[X_{i}, X_{i+1}\right]\right]=0
$$

where the $X_{i}\left(i \in \mathbb{Z}_{3}\right)$ are in $\mathfrak{g}_{x_{i}}$. In particular $\mathfrak{g}_{0}$ is an ordinary Lie algebra, and $\mathfrak{g}_{1}$ is a $\mathfrak{g}_{0}$-module with a $\mathfrak{g}_{0}$-valued symmetric bilinear form [, ] with respect to which $\mathfrak{g}_{0}$ acts as derivations:

$$
\left[X,\left[\theta_{1}, \theta_{2}\right]\right]=\left[\left[X, \theta_{1}\right], \theta_{2}\right]+\left[\theta_{1},\left[X, \theta_{2}\right]\right]
$$

for $X \in \mathfrak{g}_{0}, \theta_{i} \in \mathfrak{g}_{1}$. The basic example is the Poincare super algebra $\mathbf{p}=$ $\mathbf{p}_{0}+\mathbf{p}_{1}$, whose even part $\mathbf{p}_{0}$ is the usual Poincare algebra, and whose odd part $\mathbf{p}_{1}$ is the spinor module $S$ of the Lorentz group $\mathrm{SO}(3,1)$. (The Poincare group $P_{0}$ acts, via its quotient $\mathrm{SO}(3,1)$, on $\mathrm{p}_{1}$; this induces the infinitesimal action of $\mathbf{p}_{\mathbf{0}}$.) The pairing $\gamma: \mathbf{p}_{1} \times \mathbf{p}_{1} \rightarrow \mathbf{p}_{0}$ is Clifford multiplication, with image the translation subgroup of $\mathbf{p}_{0}$. A physicist would write everything in coordinates, so $\gamma$ becomes the collection of Pauli or Dirac matrices (depending on whether real or complex forms are used). The $N$-extended super Poincaré algebra has the same $\mathbf{p}_{0}$, but $\mathbf{p}_{1}$ is replaced by $S^{\oplus N}$, the sum of $N$ orthogonal copies of the spinors.

A supersymmetric ("SUSY") space is the super analogue of a homogeneous space for the super Poincaré algebra or its $N$-extended version. An affine example is $(V, \mathcal{O})$, where $V$ is a vector space with non-degenerate quadratic form (of signature $(3,1)$, in our case), and $\mathcal{O}$ is given in terms of the spinor module $S=S(V)$ as $C^{\infty}(V) \otimes \Lambda^{\cdot}\left(S^{\oplus N}\right)$. For more details on SUSY spaces, we refer to $[\mathrm{Be}]$, where they are defined to be super manifolds modelled on the above affine example.

An $N=1$ SUSY theory is a theory (i.e. a Lagrangian involving certain fields) with an infinitesimal action of the super Poincaré algebra. Likewise, a theory "with $N$ supersymmetries" has an action of the $N$-extended Poincaré super algebra. These theories can often, though not always, be described in terms of a super Poincaré invariant Lagrangian involving superfields on a SUSY space. The ordinary Lagrangian, on ordinary space, is recovered by Berezin integration along all the odd directions. The classic reference on supersymmetry 
is [WB]. A possibly more friendly introduction is [Fr], and the most geometrical version is $[\mathrm{Be}]$. The first two references include a study of the supersymmetric version of Yang-Mills theory.

In physics, there are two types of particles: bosons, which combine with each other freely, and fermions, whose combinations are restricted by Pauli's exclusion principle. Mathematically, the distinction is roughly this: consider a collection of $n$ particles with distinct physical properties. If $H_{i}$ is the quantum Hilbert space of states of the $i$ th particle, then the space of states of the entire collection is $\otimes_{i=1}^{n} H_{i}$. Now if instead we have $n$ identical particles, there are fewer distinct states of the ensemble: for bosons we get $\operatorname{Sym}^{n} H$, while for fermions, $\Lambda^{n} H$. In four dimensional theories, bosonic particles ordinarily live in representations of the Poincare group with integer spin, while fermions have half-integer (that is, non-integer) spin. (This is the "spin-statistics" theorem.) Most "matter" particles (electrons, protons, quarks) are fermions; photons are bosons.

At a somewhat superficial level, the motivation for introducing supersymmetry is to be able to treat the two types of particles uniformly. A complementary and deeper reason is based on the no-go theorem of Coleman and Mandula (cf. $[\mathrm{Fr}],[\mathrm{WB}]$ ). This says that the most general algebra of symmetries of a class of quantum theories satisfying some rather reasonable non-degeneracy assumptions (one of these is non-freeness, another is the existence of massive particles) is of the form $\mathbf{p}_{0} \oplus \mathfrak{g}$, where $\mathbf{p}_{0}$ is the Poincaré algebra of space-time isometries, and $\mathfrak{g}$ is the gauge algebra. The point is that this is a direct sum (of algebras), so there can be no non-trivial mixing of the two symmetries. Since symmetries are equivalent to conserved quantities, this implies for instance that any conserved quantity ("current") other than energy-momentum and angular momentum (which come from $\mathbf{p}_{0}$ ) must transform as a scalar under $\mathbf{p}_{0}$. This is unsatisfactory, since some free theories (e.g. of one real scalar field plus one real vector field, $[\mathrm{Fr}]$; such theories are not covered by the Coleman-Mandula theorem) do admit conserved vectors and tensors, which should survive under small perturbation.

The tasks of mixing the two particle types and of avoiding the ColemanMandula restriction can fortunately be accomplished simultaneously through the introduction of super Lie algebras: even elements preserve particle types, odd elements exchange them. The even part of the algebra obeys ColemanMandula, but there is room for odd symmetries and the corresponding odd, or spinorial, conserved quantities. A theorem of Haag, Sohnius and Lopuszanski classifies the possible super Lie algebras of symmetries of physically interesting theories: there are the $N$-extended super Poincare algebras, as well as some central extensions and variants including an (even) gauge algebra $\mathfrak{g}$ acting on the odd sector. 


\section{$1.5 \quad N=2$ Super Yang-Mills}

The setting for Montonen-Olive duality [MO], for the work of Seiberg-Witten [SW1, SW2], and for the development discussed in later sections, is $N=2$ supersymmetric Yang-Mills theory (SYM) in four-dimensional space. The Lagrangian for this theory is usually written in terms of super fields on an affine SUSY space [WB], making the super Poincare invariance evident. When written out explicitly in terms of its component fields, the Lagrangian is quite complicated. It starts with a purely bosonic term analogous to the usual Yang-Mills Lagrangian $\int \operatorname{Tr}(F \wedge * F)$. Additional terms, involving additional fields, are required for the $N=2$ supersymmetry. The fields involved are grouped into $N=1$ multiplets (representations of the $N=1$ super Poincare algebra), which in turn combine into $N=2$ multiplets.

Such a theory depends, of course, on the choice of a compact gauge group $G$. For a given $G$ there is the pure $N=2$ theory, analogous to Maxwell's equations in vacuo, containing only those fields and terms in the Lagrangian required for supersymmetry. There are also various theories in which some combination of additional fermionic ("matter") particles is thrown in. We will return to these shortly.

Each SYM theory has a moduli space $B$ of vacuum states (= eigenstates of the Hamiltonian or energy operator corresponding to the lowest eigenvalue). This is of course a consequence of the degeneracy, or of the gauge invariance, of the Lagrangian: a non-degenerate Lagrangian should correspond to a unique vacuum, and an ordinary degeneracy is obtained when two eigenvalues happen to coincide, so there are two (or more) independent vacua. The situation in gauge theory is that there is a continuum $B$ of independent vacua. The complex dimension of $B$ is the rank $r$ of $G$, and we can in fact describe a coordinate system $\left(u_{i}\right), i=1, \ldots, r$, on it. First, the classical analogue: the classical potential is

$$
V(\varphi)=\frac{1}{g^{2}} \operatorname{Tr}\left[\varphi, \varphi^{\dagger}\right]^{2}
$$

where $g$ is a coupling constant (a parameter in the Lagrangian), and $\varphi$ is a Higgs field--a complex field transforming as a scalar under Poincare, and in the adjoint representation of $G$. Since $\left[\varphi, \varphi^{\dagger}\right]$ is self-adjoint, the minima occur precisely when $\varphi$ commutes with $\varphi^{\dagger}$. This locus is $G$-invariant: it is the union of the $G$-orbits of elements in the Cartan subalgebra $\mathbf{t}$ of the complexified Lie algebra $\mathfrak{g}_{\mathbb{C}}$. The quotient by $G$ is thus $B=\mathbf{t} / W$, where $W$ is the Weyl group. For example, when $G=\mathrm{SU}(n)$ we have $\mathbf{t} \approx \mathbb{C}^{n-1}$, and the quotient is again $\mathbb{C}^{n-1}$, with coordinates $u_{2}=\operatorname{Tr} \varphi^{2}, \ldots, u_{n}=\operatorname{Tr} \varphi^{n}$. (The reader will no doubt recognize this as a (real) symplectic reduction: the action of $G$ on $\mathfrak{g}_{\mathbb{C}}$ is Hamiltonian with respect to the natural translation-invariant symplectic structure induced on the real vector space underlying $\mathfrak{g}_{\mathbb{C}}$ by the imaginary part of the Killing form. The moment map sends $\varphi$ to $\operatorname{Tr}\left[\varphi, \varphi^{\dagger}\right]$, and the symplectic quotient is $\mathfrak{g}_{\mathbb{C}} / / G=B=\mathbf{t} / W$. We will run into the complex analogue of symplectic quotients in the discussion of linearity in the next section.) 
Now the constraints imposed by $N=2$ supersymmetry (i.e. the difficulty of writing down a supersymmetric Lagrangian) imply that the quantum moduli space remains the same $B$, as an abstract algebraic variety, so it still has the coordinates $u_{i}$. But we will see that just about all other features of $B$ change in going from the classical to the quantum: for example, this applies to the Kähler metric on it, the locus of singularities (of the metric), the flat structure, and the relation of the $u$ coordinates to the natural fields in the theory.

A central quantity in any SYM theory is the electric charge $a$, which is the vector of eigenvalues of the Higgs field $\varphi$. It lives in the Cartan, t. For SU(2) this is a complex number, as is $u$. We can take $\varphi$ to have eigenvalues $\pm a / 2$, and then $u=\operatorname{Tr} \varphi^{2}=\frac{a^{2}}{2}$, in the classical theory. In the quantum theory, this relationship still holds approximately as $u \rightarrow \infty$, by the asymptotic freedom of the theory. But for finite $u$ we will see that the relationship is very different.

A crucial property of these theories is that their low-energy (read: realworld observable) behavior can be described in terms of only a finite number of parameters-the coordinates $u_{i}$ on the quantum moduli space $B$. This description is via a "low energy effective Lagrangian" $\mathcal{L}_{\text {eff }}$, obtained from the full Lagrangian through a process of averaging over all heavy degrees of freedom. So all measurable quantities become (locally) functions on $B$. In particular, this applies to the components $a_{i}=a_{i}(u)$ of the electric charge. Supersymmetry implies that these functions are holomorphic, and we will use them as alternative local coordinates on $B$.

While the existence of $\mathcal{L}_{\text {eff }}$ is guaranteed by the general theory, its actual computation can be extremely difficult. The supersymmetry allows $\mathcal{L}_{\text {eff }}$ to be expressed locally in terms of a single holomorphic function $\mathcal{F}=\mathcal{F}(a)$, the prepotential. (This is due to Seiberg, cf. [SW1], (2.7) and (2.11). Note that we need $\mathcal{F}$ as a function of $a$, not of $u$.) All other quantities in the theory can then be expressed in terms of $\mathcal{F}$ and the coordinates $a$. This includes the vector

$$
a^{D}=\frac{d \mathfrak{F}}{d a}
$$

whose physical significance will appear shortly; the matrix

$$
\tau(a)=\frac{d^{2} \mathcal{F}}{d a^{2}}=\frac{d a^{D}}{d a}
$$

which is a complexified coupling constant (for $\mathrm{SU}(2)$, the low-energy effective values of the real coupling constant $g$ (encountered in the potential $V(\varphi)$ ) and of a phase angle $\theta$ are given by $\tau=\frac{\theta}{2 \pi}+\frac{4 \pi i}{g^{2}}$. In particular, $\mathcal{F}(a)$ must be such that $\operatorname{Im}(\tau)>0)$; the Kähler metric

$$
d s^{2}=\operatorname{Im}(\tau d a d \bar{a})=\operatorname{Im}\left(d a^{D} d \bar{a}\right)
$$

which was mentioned above; and its Kähler potential

$$
K=K(a, \bar{a})=\operatorname{Im}\left(\sum_{i} a_{i}^{D} \bar{a}_{i}\right)=\operatorname{Im}\left(\sum_{i} \frac{d \mathcal{F}}{d a_{i}} \bar{a}_{i}\right) .
$$


The effective Lagrangian itself is given as a sum of kinetic and potential parts, each a Berezin integral over an $N=1$ SUSY affine space of dimensions $(4,4)$ and $(4,2)$, respectively:

$$
\mathcal{L}_{\mathrm{eff}}=\frac{1}{4 \pi} \int K(\Phi, \bar{\Phi}) d^{4} \eta+\frac{1}{8 \pi} \operatorname{Im} \int \tau(\Phi)|W|^{2} d^{2} \eta
$$

Here $\Phi$ and $W$ are the two $N=1$ multiplets which together make up the $N=2$ multiplet containing the Higgs field $\varphi$. ( $\varphi$ and a fermion $\psi$ are the fields in the "chiral multiplet" $\Phi$. The "vector multiplet" $W$ contains another fermion $\lambda$ and the gauge field $A$. It can be thought of as the field strength, $N=1$ super analogue of the curvature $F$ in ordinary YM.) The integrands involve an analytic function $(\tau)$ or the real part of one $(K)$; this function is to be applied formally to the super fields $\Phi, W$ and their conjugates. The integration over the four (or two) odd space directions $\eta$ is interpreted via Berezin's recipe as a derivative, or as the reading off of the relevant coefficients. (The second term is actually written as an integral over a $(4,2)$-dimensional "chiral" quotient of the full $(4,4)$-dimensional SUSY space, in which two of the odd directions have been reduced.)

What is missing is an explicit expression for $\mathcal{F}$. Traditionally, $\mathcal{F}$ was written as an infinite sum involving its classical ("tree level") value, the one-loop correction which is logarithmic in $a$ (higher loop contributions were known to vanish), and an infinite sum of mysterious instanton corrections involving negative powers of $a$. The breakthrough came when Seiberg and Witten succeeded, in [SW1], in computing $\mathcal{F}$ (and hence $\mathcal{L}_{\text {eff }}, d s^{2}$, etc.) exactly, for the pure $N=2$ theory with $G=\mathrm{SU}(2)$. Their solution is based on the global properties of the theory.

\subsection{Duality}

The original conjecture of Montonen and Olive [MO] was that the low energy theory should be invariant under a duality exchanging electric and magnetic charges, and also exchanging the coupling constant $g$ with its inverse. In other words: electric charge in the strongly coupled theory (large $g$ ) behaves like magnetic charge at weak coupling, and vice versa. Seiberg and Witten found that a modified version of this duality holds in pure $N=2 \mathrm{SYM}$ for SU(2). They applied the super version of the Hodge $*$ operator to the Lagrangian $\mathcal{L}_{\text {eff }}$, added some variables (Lagrange multipliers) and integrated out others, and ended up with a new Lagrangian $\mathcal{L}_{\text {eff }}^{D}$ which is equivalent to the original, and has the same form, but in terms of a new quantity $a^{D}:=\frac{d \mathcal{F}}{d a}$ :

$$
\mathcal{L}_{\text {eff }}^{D}\left(a^{D}\right)=\mathcal{L}_{\text {eff }}(a)
$$

The same transformation sends $a^{D}$ to $-a=-\frac{d \mathcal{F}}{d a^{D}}$. (This must be so, since the first term in $\mathcal{L}_{\text {eff }}$ changes sign when $a, a^{D}$ are simply exchanged.) The physical meaning of $a^{D}$ is therefore that of the dual, or magnetic, charge. As to the 
coupling constants, the defining relations

$$
\tau=\frac{d a^{D}}{d a}, \quad \tau^{D}=\frac{d(-a)}{d a^{D}}
$$

together with the chain rule, imply that the actual duality transformation is

$$
\tau^{D}=\tau^{D}\left(a^{D}\right)=-\tau(a)^{-1} .
$$

This specializes to the Montonen-Olive transformation

$$
g^{D}=g^{-1}
$$

when $\theta=0$, but not otherwise.

There is another, more elementary, transformation which preserves the Lagrangian: the $\theta$ angle is defined only modulo $2 \pi \mathbb{Z}$, so $\tau$ is defined modulo $\mathbb{Z}$. There is therefore a transformation which fixes $a$ and sends $\tau \mapsto \tau+1$. Since $\tau=\frac{d a^{D}}{d a}$, this requires that $a^{D}$ goes to $a^{D}+a$.

The two transformations can be represented by the matrices

$$
S=\left(\begin{array}{cc}
0 & 1 \\
-1 & 0
\end{array}\right) \quad T=\left(\begin{array}{ll}
1 & 1 \\
0 & 1
\end{array}\right)
$$

acting linearly on the 2-vector $\left(\begin{array}{c}a^{D} \\ a\end{array}\right)$ and fractional-linearly on $\tau$. Since $S$ and $T$ generate $\mathrm{SL}(2, \mathbb{Z})$, we see that the theory has an $\mathrm{SL}(2, \mathbb{Z})$ action. (For other groups, $\mathrm{SL}(2, \mathbb{Z})$ is replaced by a subgroup of finite index in $\operatorname{Sp}(2 r, \mathbb{Z})$.)

\subsection{The Seiberg-Witten solution}

The foregoing suggests that what lives intrinsically over a generic $u \in B$ is not the electric charge $a(u)$ but the unimodular lattice $\mathbb{Z} a(u)+\mathbb{Z} a^{D}(u)$ of all charges (electric, magnetic, and "dyonic", or mixed). As $u$ varies, we get a $\mathbb{Z}^{2}$ local system $V$ over $B$ minus some singular locus, together with a homomorphism to (the trivial bundle with fiber) $\mathbb{C}$. Dually, this homomorphism is equivalent to a holomorphic section $\left(\begin{array}{c}a^{D} \\ a\end{array}\right)$ of the vector bundle $V_{\mathbb{C}}$ (i.e. of the sheaf $V \otimes \mathcal{O}_{B \backslash \text { sing }}$ ). The plan is to identify the local system (variation of Hodge structure, really) $V$, and then to fix the section $\left(\begin{array}{c}a^{D} \\ a\end{array}\right)$, which in turn will determine the prepotential $\mathcal{F}$ (up to constant).

Seiberg and Witten identify $V$ by finding its monodromy. Near $u=\infty$ we know that $u \sim \frac{1}{2} a^{2}$, so the monodromy around $\infty$ sends $a \mapsto-a$. The known non-trivial logarithmic (one-loop) term in the perturbative expansion of $\mathcal{F}$ then fixes the shift in $a^{D}$ : the monodromy is

$$
M_{\infty}=\left(\begin{array}{cc}
-1 & 2 \\
0 & -1
\end{array}\right)
$$

In particular, the local system is non trivial, so $\tau$ is a non-constant holomorphic function on the universal cover of $B \backslash$ sing, with values in $\mathbb{H}$. The singular 
locus must therefore contain at least two points (in addition to $\infty$ ). From a physical argument, Seiberg and Witten deduce that the system is as simple as possible: exactly three singularities, which can be relocated to $u= \pm 1$ and $\infty$ by an automorphism. Duality instructs that the finite monodromies should be conjugate to $-M_{\infty}$. Seiberg and Witten label things so that $a^{D}$ vanishes at $u=1$, yielding the monodromy

$$
M_{1}=\left(\begin{array}{cc}
1 & 0 \\
-2 & 1
\end{array}\right)
$$

which then determines $M_{-1}$.

The monodromies generate the level-2 congruence subgroup $\Gamma(2) \subset \operatorname{SL}(2, \mathbb{Z})$, and the local system itself can now be identified with the fiber cohomology of the universal level-2 elliptic curve

$$
E_{u}: y^{2}=(x+1)(x-1)(x-u)
$$

over the $u$-line $\mathbb{C} \backslash\{ \pm 1\}$.

The complexification $V_{\mathbb{C}}$ has a global trivialization in terms of the holomorphic 1-form $\lambda_{1}=\frac{d x}{y}$ and the residueless meromorphic form $\lambda_{2}=\frac{x d x}{y}$. We can also fix an integral homology basis, say $\gamma=$ loop around the branch points 0,1 , and $\gamma^{D}=$ loop around $1, u$. To determine $a(u)$ and $a^{D}(u)$, we use the condition that $\operatorname{Im}(\tau)>0$, where $\tau(u):=\frac{d a^{D} / d u}{d a / d u}$. One geometric solution is to take $\tau(u)$ as the period

$$
\tau_{u}:=\frac{\oint_{\gamma^{D}} \lambda_{1}}{\oint_{\gamma} \lambda_{1}}
$$

of the elliptic curve $E_{u}$; rigidity implies that this is in fact the only solution. We then find that, up to a multiplicative constant, $a$ and $a^{D}$ are the periods over $\gamma, \gamma^{D}$ of the meromorphic 1-form

$$
\lambda=\frac{y d x}{x^{2}-1}=\frac{(x-u) d x}{y}=\lambda_{2}-u \lambda_{1} .
$$

\subsection{Adding matter}

In a quantum field theory, a matter particle is represented by a fermion field $\psi$ which, in the case of a gauge theory, needs to be in some representation of $G$. It is added (or: coupled) to the theory by adding to the YM Lagrangian a term corresponding to the Lagrangian of the free particle (this includes a parameter, the particle mass $m$ ), and another term corresponding to its interaction with the YM field. In SYM the field needs to be in a representation $\rho$ of $G$, tensored with an $N=2$ multiplet, to preserve the SUSY. (The relevant multiplet, which is built from the fermion $\psi$, is called a hypermultiplet; we have already encountered the other basic type, the $N=2$ vector multiplet, which contained the scalar Higgs field $\varphi$.) If the number of particles added is not too large, the 
theory remains asymptotically free $(\beta<0)$ or becomes scale invariant $(\beta=0)$, and in either case retains many properties of the pure SYM theory. There still is an $r$-dimensional moduli space $B$. At $u \in B$ there are $r$ electric charges $a_{i}(u)$ and $r$ dual, magnetic, charges, $a_{i}^{D}(u)$, each charge living in the Cartan $\mathbf{t} \approx \mathbb{C}^{r}$ as before. Duality is modified somewhat: instead of $\operatorname{Sp}(2 r, \mathbb{Z})$ transformations acting on $\left(\begin{array}{c}a^{D} \\ a\end{array}\right)$, one gets affine symplectic transformations of the form

$$
\left(\begin{array}{c}
a^{D} \\
a
\end{array}\right) \longmapsto R\left(\begin{array}{c}
a^{D} \\
a
\end{array}\right)+\sum_{i=1}^{N_{f}} m_{i}\left(\begin{array}{c}
n_{i}^{D} \\
n_{i}
\end{array}\right)
$$

where $R \in \operatorname{Sp}(2 r, \mathbb{Z})$, the $m_{i}$ are the masses of the $N_{f}$ particles added, and $n_{i}$, $n_{i}^{D}$ are integral $r \times r$ matrices. ( $N_{f}$ " stands for number of flavors. The number of colors $N_{c}$ is the dimension of the representation space of $\rho$. The terminology comes from quantum chromodynamics (QCD), a gauge theory with $G=\mathrm{SU}(3)$.)

It is reasonable to guess that this structure can be implemented by a family of $r$-dimensional abelian varieties $A_{u}$ over $u \in B \backslash$ (sing): The $r \times 2 r$ period matrix of $A_{u}$ in some integral homology basis $\gamma^{D}, \gamma$ is $\left(\frac{d a^{D}(u)}{d u}, \frac{d a(u)}{d u}\right)$. (The polarization on the complex torus corresponding to the lattice generated by columns of this matrix is given by the Dirac quantization condition.) On the total space of the family there is a meromorphic 1-form $\lambda$, the Seiberg-Witten differential. There are $N_{f}$ divisors $D_{i}$ along which $\lambda$ acquires a pole with constant residue $\frac{m_{i}}{2 \pi i}$. The charges $a^{D}, a$ can then be recovered as the periods of $\lambda$ over $\gamma^{D}, \gamma$. These have precisely the ambiguity needed: monodromy of the cycles $\gamma^{D}, \gamma$ acts as $\operatorname{Sp}(2 r, \mathbb{Z})$, while moving such a cycle across the divisor $D_{i}$ changes the period by an integer multiple of $m_{i}$. The masses $m_{i}$ acquire a cohomological interpretation: the 2 -form $\sigma=d \lambda$ is holomorphic (in fact, it is a holomorphic symplectic form) on the total space, and its de Rham cohomology class can be expressed in terms of the fundamental classes of the $D_{i}$ :

$$
[\sigma]=\sum m_{i}\left[D_{i}\right]
$$

In particular, as we vary the masses, we obtain a family of algebraic symplectic manifolds in which the symplectic class varies linearly in the parameters.

In the case of scale-invariant theories $(\beta=0)$, the theory and therefore also its geometric model depend on one parameter in addition to the masses. This is $\tau_{c l}$, the classical limit $(u \rightarrow \infty)$ of the quantum coupling constant $\tau=\tau(u)$. In asymptotically free theories $(\beta<0)$ we have

$$
\lim _{u \rightarrow \infty} \tau(u)=\infty
$$

and instead of $\tau$ the theory depends on a scale parameter $\Lambda$. In the [SW1] solution, this $\Lambda$ is set to 1 for convenience.

In [SW2] Seiberg and Witten show that the above geometric model indeed leads to an exact solution of the various SU(2) SYM theories with matter. For each of these, the solution requires a family parametrized by the vector 
$m=\left(m_{i}\right)$ of masses (and by the additional parameter $\tau$ or $\Lambda$, which we omit from notation), of elliptic surfaces

$$
X_{m} \rightarrow B \approx \mathbb{C}
$$

with meromorphic 1 -forms $\lambda_{m}$ whose residues equal $\frac{m}{2 \pi i}$. The solution satisfies numerous consistency checks (the way one theory is known to flow to another in the limit where some parameter, such as a mass, goes to 0 or $\infty$, is reflected in corresponding degenerations of one geometric model to another) and has all the internal symmetry of each of the physical theories.

The number of particles which can be added to a SYM theory is determined as follows. The Killing form on the reductive Lie algebra $\mathfrak{g}$ determines an element $c_{2}$ in the center of the universal enveloping algebra $U(\mathfrak{g})$. By Schur's lemma, $c_{2}$ acts on the total space of each irreducible representation $\rho$ as multiplication by some scalar $c_{2}(\rho)$. (This $c_{2}$ is closely related to the second chern class of a bundle: Let $V, V^{\prime}$ be two vector bundles on a surface associated via representations $\rho, \rho^{\prime}$ to the same $G$-bundle. Then

$$
\frac{c_{2}\left(\rho^{\prime}\right)}{c_{2}(\rho)}=\frac{c_{2}\left(V^{\prime}\right)}{c_{2}(V)}
$$

where $c_{2}$ on the right denotes Chern classes. This can be used to give another definition of $c_{2}$ of a representation.) In any case, the $\beta$ function of the SYM theory with particles in representations $\rho_{i}, i=1, \ldots, N_{f}$, is

$$
\beta=c_{2}(\mathrm{ad})-\sum_{i} c_{2}\left(\rho_{i}\right)
$$

where ad is the adjoint representation. For the classical algebras we can adjust the scaling of the Killing form so that $c_{2}(F)=1$ where $F$ is the fundamental representation. With this normalization, $c_{2}(\mathrm{ad})=2 h_{\mathfrak{g}}^{\vee}$, where $h_{\mathfrak{g}}^{\vee}$ is the dual Coxeter number. For $\mathrm{SU}(n)$ we have $h_{\mathfrak{g}}^{\vee}=n$, so the $\mathrm{SU}(2)$ theory, for example, remains asymptotically free with $N_{f}=0,1,2$ or 3 particles in the fundamental representation $F$, and becomes scale invariant for $N_{f}=4$ fundamental particles or for one particle in the adjoint representation. These are precisely the cases studied and solved in [SW2].

It is natural to ask whether the four dimensional, $N=2$ SYM theory with arbitrary gauge groups $G$ and collection of particles $\rho_{i}$ with $\beta \leq 0$ can be similarly solved in geometric terms via families of abelian varieties and meromorphic one-forms. This question will occupy us in the remainder of this work.

\section{$2 \quad$ Why integrable systems}

\subsection{Algebraically integrable systems}

An algebraically integrable system consists of a complex algebraic manifold $X$, an everywhere non-degenerate, closed holomorphic $(2,0)$-form $\sigma$ on $X$, and a 
morphism $\pi: X \rightarrow B$ whose general fiber is a (polarized) abelian subvariety $X_{b}:=\pi^{-1}(b)$ which is Lagrangian with respect to $\sigma$. In practice, the object which is naturally given is often an affine open $X_{0} \subset X$, the fibers being affine subsets of abelian varieties. This is a straightforward algebraic analogue of the (real, $C^{\infty}$ ) notion of integrable system in classical mechanics. As there, any function $H$ ("Hamiltonian") on the base $B$ determines a 1-form $d \pi^{*} H$ on $X$, and when contracted with the symplectic form $\sigma$ it gives a vector field $v_{H}$ on $X$, tangent to the fibers of $\pi$. The pullbacks $\pi^{*} H_{1}, \pi^{*} H_{2}$ of two functions on $B$ Poisson-commute, and in particular the corresponding vector fields commute.

Locally on the base of an algebraically integrable system there is a natural holomorphic flat structure, depending on a discrete choice. The fiber homology $H_{1}\left(X_{b}, \mathbb{Z}\right)$ has a non-degenerate skew-symmetric pairing (induced by the polarization). The choice involved is that of a Lagrangian subspace $V_{\mathbb{Z}}^{*}$ for this pairing. This is often achieved by specifying a symplectic basis $\alpha_{1}, \ldots, \alpha_{g}, \beta_{1}, \ldots, \beta_{g}$ of $H_{1}\left(X_{b}, \mathbb{Z}\right)$, and taking the subspace $V_{\mathbb{Z}}^{*}$ spanned by the $\alpha$ cycles. This choice needs to be made for one $b \in B$, and (by Gauss-Manin) is propagated to nearby fibers. Integration over these $\alpha$-cycles gives a local trivialization of the relative cotangent bundle $\pi_{*} \omega_{X}$, and the symplectic form $\sigma$ converts this to a flat structure on (the tangent bundle of) $B$, modelled on $V:=\operatorname{Hom}\left(V_{\mathbb{Z}}^{*}, \mathbb{C}\right.$ ).

Locally on the flat base $B$, we can ask what kind of data is required to specify an algebraically integrable system inducing the given flat structure. We think of the family of Abelian varieties, plus choice of $\alpha$ and $\beta$ cycles, as specified by its period map

$$
p: B \rightarrow \mathbb{H}_{g}
$$

to the Siegel half space $\mathbb{H}_{g}$ of $g \times g$ symmetric complex matrices with positive imaginary part. The total space $\pi: X \rightarrow B$ is then retrieved as pullback of the universal abelian variety $X_{g} \rightarrow \mathbb{H}_{g}$. The question then is to understand the condition on $p$ for there to be a symplectic form on $X$ for which $\pi$ is a Lagrangian fibration. The fixing of the flat structure is achieved by thinking of $\mathbb{H}_{g}$ as open subset of $\operatorname{Sym}^{2} V^{*}$, where $V$ is the tangent space to $B$ (at any $b \in B$ ). The derivative of the period map is

$$
d p_{b} \in \operatorname{Hom}\left(V, \operatorname{Sym}^{2} V^{*}\right)=V^{*} \otimes \operatorname{Sym}^{2} V^{*} .
$$

A straightforward computation done in [DM2] shows that the condition is that $d p_{b}$ lives in the subspace $\operatorname{Sym}^{3} V^{*}$, for all $b \in B$. So an integrable system with base $B$ is specified by a field of cubics on the tangent bundle of $B$.

This field of cubics is subject, of course, to a strong integrability condition. In flat local coordinates $z_{i}$ on $B$, let $\left(p_{i j}(z)\right)$ be the period matrix and $\left(c_{i j k}(z)\right)$ the field of cubics. Then equality of mixed partials

$$
\frac{\partial p_{i j}}{\partial z_{k}}=c_{i j k}=\frac{\partial p_{i k}}{\partial z_{j}}
$$

implies the existence of functions $w_{i}$ (locally) on $B$ such that

$$
p_{i j}=\frac{\partial w_{i}}{\partial z_{j}} .
$$


These again have symmetric partials,

$$
\frac{\partial w_{i}}{\partial z_{j}}=p_{i j}=\frac{\partial w_{j}}{\partial z_{i}}
$$

so there is a single function $\mathcal{F}$ on $B$, called the prepotential, satisfying:

$$
\begin{aligned}
w_{i} & =\frac{\partial \mathcal{F}}{\partial z_{i}} \\
p_{i j} & =\frac{\partial^{2} \mathcal{F}}{\partial z_{i} \partial z_{j}} \\
c_{i j k} & =\frac{\partial^{3} \mathcal{F}}{\partial z_{i} \partial z_{j} \partial z_{k}}
\end{aligned}
$$

The prepotential determines also a Kähler potential on $B$

$$
K:=\operatorname{Im}\left(\sum_{i} w_{i} \bar{z}_{i}\right)=\operatorname{Im}\left(\sum_{i} \frac{\partial \mathcal{F}}{\partial z_{i}} \bar{z}_{i}\right)
$$

and its associated Kähler form

$$
\frac{i}{2} \partial \bar{\partial} K=\frac{i}{2} \sum_{i, j} \operatorname{Im}\left(p_{i j}\right) d z_{i} \wedge d \bar{z}_{j} .
$$

Equivalently, this Kähler $(1,1)$-form on $B$ is obtained by integration over the $g$-dimensional fibers $X_{b}$ of the $(g+1, g+1)$-form $\sigma \wedge \bar{\sigma} \wedge t^{g-1}$ on $X$, where $t$ is any $(1,1)$-form on $X$ whose restrictions to fibers give the polarization.

Conversely, given $B$ with coordinates $z_{i}$ and a holomorphic function $\mathcal{F}$, the above formulas produce an algebraically integrable system over the open subset of $B$ where $\operatorname{Im}\left(\frac{\partial^{2} \mathcal{F}}{\partial z_{i} \partial z_{j}}\right)>0$ : the family of abelian varieties is determined by its periods $p_{i j}$, and the symplectic form is given by the cubic $c_{i j k}$. The slightly weaker notion of an analytically integrable system (just replace abelian varieties by polarized complex tori) is recovered over the open subset where $\operatorname{Im}\left(\frac{\partial^{2} \mathcal{F}}{\partial z_{i} \partial z_{j}}\right)$ is invertible, cf. [DM2]. The Kähler "metric" in this case is still non-degenerate, but possibly indefinite.

We note that the $w_{i}$ play a role dual to that of the $z_{i}$ : the $z_{i}$ give the flat structure on $B$ determined by integration over the $\alpha$ cycles, while the $w_{i}$ give the flat structure determined by integration over the $\beta$ cycles. We also note that, up to this point, the $z_{i}$ as well as the $w_{i}$ have been determined only up to arbitrary additive constants. In other words, what we have are the vector fields $\partial / \partial z_{i}, \partial / \partial w_{i}$, or the 1 -forms $d z_{i}, d w_{i}$. We will discuss below the additional choice used in the SW setup to fix these additive constants.

Similar structures have arisen in various more specialized contexts, e.g. in the WDVV equations of conformal field theory and on moduli spaces of CalabiYau threefolds, as well as in the Seiberg-Witten setup described in $\S 1$. They 
often go under the title of "special geometry". The point is that these specialized contexts are not really needed: the structure of special geometry on $B$ (consisting of a covering by open subsets on which an appropriate prepotential, dual flat holomorphic coordinate systems, and a Kähler metric are defined), arises naturally on the base of any algebraically integrable system. The converse is also true, locally, as we have just seen. Globally, though, there is an integrability constraint: the monodromy action (say on the coordinates $\left.z_{i}, w_{i}\right)$ in an integrable system involves integral symplectic transformations plus (complex) translations, while special geometry allows the symplectic transformation to be real instead of integral. We refer the reader to $[F]$ for a very clear differential-geometric discussion of special geometry and its relationship to integrable systems.

\subsection{Seiberg-Witten Differentials}

In the Seiberg-Witten picture of super Yang-Mills, there are the charges $a_{i}$ and $a_{i}^{D}$ which are described locally as holomorphic functions on the quantum moduli space $B$. There is less ambiguity in choosing these $a_{i}, a_{i}^{D}$ than in the coordinates $z_{i}, w_{i}$ on the base of an algebraically integrable system: the group of their linear combinations $\sum n_{i} a_{i}+n_{i}^{D} a_{i}^{D}$ (with integer coefficients $n_{i}$ ) should be uniquely determined in the pure SYM case, and determined modulo the masses of the added particles in general. A natural way to get functions with precisely such ambiguity on the base of an integrable system $\pi: X \rightarrow B$ is to choose a meromorphic differential 1 -form $\lambda$ on $X$ and let $a_{i}, a_{i}^{D}$ be its periods over a set of 1-cycles representing $\alpha_{i}, \beta_{i}$ respectively and avoiding the poles of $\lambda$. We want $a_{i}, a_{i}^{D}$ to be a possible choice of the coordinates $z_{i}, w_{i}$, which are defined up to translation (once we have fixed the $\alpha_{i}, \beta_{i}$ ). In other words, we want

$$
d a_{i}=d z_{i}, \quad d a_{i}^{D}=d w_{i} .
$$

Since $d z_{i}, d w_{i}$ were defined as the contraction of $\alpha_{i}, \beta_{i}$ respectively with the symplectic form $\sigma$, the condition becomes simply:

$$
d \lambda=\sigma .
$$

The behavior of the singularities of $\lambda$ is constrained by this condition. When restricted to a general fiber $X_{b}, \lambda$ will have poles along the union of some irreducible divisors $D_{b, j}$. One constraint is that the residue $\operatorname{Res}_{j}$ of $\lambda$ along $D_{b, j}$ is (locally in $B$ ) independent of $b$. A Seiberg-Witten differential is a meromorphic 1 -form $\lambda$ satisfying $d \lambda=\sigma$ and $\operatorname{Res}_{j}=m_{j}$, the mass of the $j$-th particle. On an algebraically integrable system with a specified Seiberg-Witten differential, the local coordinates $z_{i}, w_{i}$ can be specified as the charges $a_{i}, a_{i}^{D}$, with just the right ambiguity.

One way to describe an integrable system (though by no means the only way!) is in terms of a family $C \rightarrow B$ of spectral curves $C_{b}$. In the simplest situation, the abelian varieties $X_{b}$ are just the Jacobians $J\left(C_{b}\right)$. More generally, 
$X_{b}$ may be realized as the Prym of $C_{b}$ with respect to an involution, or as the generalized Prym with respect to a correspondence, cf. [D1]. An example of such a system, due to Mukai [Mu], is obtained from the Jacobians of a complete linear system $C \rightarrow B$ of curves on a symplectic surface $(S, \bar{\sigma})$. The symplectic form $\sigma$ on $X$ then corresponds via Abel-Jacobi to the pullback to $C$ of $\bar{\sigma}$. Mukai studied the case where $S$ is a K3 surface. Taking $S=T^{*} E$ to be the cotangent bundle of some curve $E$ yields Hitchin's system on $E$. One obtains many more examples by allowing $S$ to have a symplectic form $\bar{\sigma}_{0}$ defined only on an open subset $S_{0} \subset S$, and imposing appropriate restrictions on the intersection of the spectral curves $C_{b}$ with $S-S_{0}$. For example, we can take $S$ to be the total space of the line bundle $\omega_{E}(D)$ for some effective divisor $D$ on $E$, to recover Markman's system [Mn] parametrizing meromorphic Higgs bundles on $E$. Note that this $S$ has a natural meromorphic 1-form $\bar{\lambda}$, given away from $D$ (i.e. where $S$ can be identified with $T^{*} E$ ) as the action 1 -form $\bar{\lambda}=p d q$, whose differential is $d \bar{\lambda}=\bar{\sigma}_{0}$. It follows that the "tautological" 1 -form on $C$ (pullback of $\bar{\lambda}$ ) induces the Seiberg-Witten differential $\lambda$ on the integrable system $X=J(C / B) \rightarrow B$. The system discussed in $\S 3$ is of this form.

\subsection{Linearity: complexified Duistermaat-Heckman}

One feature of the Seiberg-Witten picture which we have not yet discussed is the linear dependence of the symplectic form on the particle masses. From one point of view, this linearity follows from existence of the Seiberg-Witten differential: the equation $\sigma=d \lambda$ implies that the cohomology class of $\sigma$ is a fixed linear combination of the residues of $\lambda$, which are just the masses. On the other hand, linearity can also be interpreted as arising from a complex analogue of the theorem of Duistermaat-Heckman [DH].

Let $(X, \sigma)$ be a complex symplectic manifold (i.e. the symplectic form $\sigma$ is holomorphic, of type $(2,0)$ ) equipped with a Hamiltonian action of a complex reductive group $G$. The Hamiltonian property of the action means that there is a moment map $\mu: X \rightarrow \mathfrak{g}^{*}$ to the dual of the Lie algebra. Ignoring bad loci, we will assume (or pretend) that there is a good non-singular quotient $X / G$. Let $G_{a} \subset G$ be the stabilizer of $a \in \mathfrak{g}^{*}$ under the coadjoint action. The symplectic quotient $X_{a}$ (or $X / /{ }_{a} G$ ) is defined as

$$
X_{a}:=\mu^{-1}(a) / G_{a}=\mu^{-1}\left(\mathcal{O}_{a}\right) / G,
$$

where $\mathcal{O}_{a}$ is the (coadjoint) orbit of $a$. It is again a complex symplectic manifold, with form $\sigma_{a}$. As $a$ ranges over orbits of a fixed "type" in $\mathfrak{g}^{*}$, the topology of $X_{a}$ remains locally constant, so cohomologies of nearby $X_{a}$ 's of fixed type can be identified. The complex Duistermaat-Heckman theorem says that, under this identification, the cohomology class $\left[\sigma_{a}\right] \in H^{2}\left(X_{a}\right)$ varies linearly with $a$.

Consider first the abelian case where $G=T$ is a torus $T=\operatorname{Hom}\left(\Lambda, \mathbb{C}^{*}\right)$. The moment map then factors

$$
X \rightarrow X / T \rightarrow \mathbf{t}^{*},
$$


and we assume both maps are bundles, at least over some open $\mathbf{t}_{0}^{*} \subset \mathbf{t}^{*}$. The $T$-bundle $X \rightarrow X / T$ has first chern class

$$
c_{1} \in H^{2}\left(X / T, \Lambda^{*}\right) .
$$

For each $a$ in $\mathbf{t}_{0}^{*}$, this restricts to a class (still denoted by $c_{1}$ ) in $H^{2}\left(X_{a}, \Lambda^{*}\right)$. The theorem in this case says that for $a, a_{0} \in \mathbf{t}_{0}^{*}=\Lambda \otimes \mathbb{C}$,

$$
\left[\sigma_{a}\right]-\left[\sigma_{a_{0}}\right]=\left\langle c_{1}, a-a_{0}\right\rangle
$$

Because of possible monodromy of the fibers of $X / T$ over $\mathbf{t}_{0}^{*}$, both sides may be multivalued, so we should think of the linearity as being local in $a$.

Next, let $G$ be an arbitrary reductive group, and consider reduction at a regular semisimple element $a$. This means that the stabilizer $G_{a}$ is a maximal torus $T \subset G$. The coadjoint orbits of semisimple elements are parametrized by the quotient $\mathbf{t}^{*} / W$ of the dual Cartan by the Weyl group. Let $\mathfrak{g}_{0}^{*}, \mathbf{t}_{0}^{*}$ denote the open subsets of $\mathfrak{g}^{*}, \mathbf{t}^{*}$ respectively parametrizing regular semisimple elements. The parameter space for regular semisimple coadjoint orbits is then

$$
\mathbf{t}_{0}^{*} / W \approx \mathfrak{g}_{0}^{*} / G
$$

The moment map in this nonabelian situation no longer factors. The closest we get to factorization is the commutative diagram:

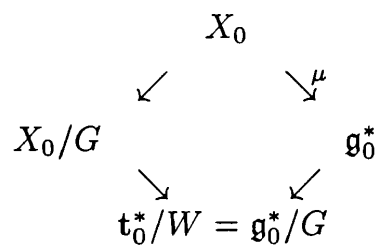

with $X_{0}:=\mu^{-1}\left(\mathfrak{g}_{0}^{*}\right)$. Somewhat surprisingly, it is easy to reduce this situation to the abelian case, by considering $Y:=\mu^{-1}\left(\mathbf{t}^{*}\right)$. The symplectic form of $X$ restricts to a symplectic form on $Y$, and the $T$-action on $Y$ is Hamiltonian with respect to this form, so there is a moment map $\mu_{T}: Y \rightarrow \mathbf{t}^{*}$ compatible with $\mu$ via the inclusions $Y \subset X$ and $\mathbf{t}^{*} \subset \mathfrak{g}^{*}$. Now for $a \in \mathbf{t}_{0}^{*}$ there is an obvious identification of symplectic manifolds $X / /{ }_{a} G \approx Y / /{ }_{a} T$, so the dependence of the symplectic class $\left[\sigma_{a}\right]$ of $X / /{ }_{a} G$ on $a \in t_{0}^{*}$ is again locally linear. (In the original case with $G$ compact, the multivaluedness is replaced by discontinuities across walls, resulting in $\left[\sigma_{a}\right]$ being linear on each Weyl chamber).

What happens when $a$ is allowed to degenerate? If $G$ is compact, $a$ remains semisimple (though not regular), and there is a fairly obvious linearity for all such $a$ in a given open face $\mathbf{f}_{0}$ of the Weyl chamber, i.e. a face $\mathbf{f}$ minus its proper subfaces. In the complex reductive case, there are more possibilities: the most natural orbit which corresponds to an $a$ in a complexified open face $\mathbf{f}_{0}$ consists of elements which are not semisimple but regular, in the sense that their centralizers have the minimal possible dimension. The closure of this 
orbit consists of a finite number of other orbits. The smallest of these orbits (the unique closed orbit, which is in the closure of each of the others) consists of the semisimple elements. Although we will not encounter non-regular elements in the integrable systems we need in the following section, it seems worthwhile to point out that linearity is indeed a general phenomenon applicable to all strata.

We identify $\mathfrak{g}, \mathbf{t}$ with $\mathfrak{g}^{*}, \mathbf{t}^{*}$ via an invariant form. Consider an arbitrary stratum $S \subset \mathfrak{g}$ corresponding to a face $\mathbf{f}_{0} \subset \mathbf{f} \subset \mathbf{t} . S$ is a bundle over (a finite quotient of the complexified) $\mathbf{f}_{0}$, and the fibers are (co)adjoint orbits of a fixed type. This bundle has local sections, which can be taken of the form $n+\mathbf{f}_{0}$, where $n$ is some fixed nilpotent in the reductive subalgebra $\mathbf{l}=\mathbf{l}_{\mathbf{f}}$ ( $=$ common centralizer of all elements of $\mathbf{f}_{0}$ ). All elements $a=n+t$ of this section have the same centralizer $\mathbf{c}$ in $\mathfrak{g}$. ( $\mathbf{c}$ is the centralizer in $\mathbf{l}$ of $n$ ). Since $\mathbf{f}$ is the center of $\mathbf{l}$, it is contained in $\mathbf{c}$, which actually decomposes as $\mathbf{c}=\mathbf{f}+\mathbf{d}$ with $\mathbf{d}$ semisimple. Let $D \subset G$ be the subgroup corresponding to $\mathbf{d}$. The reduction to the abelian case now proceeds as follows: We observe that $\mu^{-1}\left(n+\mathbf{f}_{0}\right) / D$ is symplectic, and has a residual Hamiltonian action of the subtorus $F \subset T$ whose algebra is $\mathbf{f}$. Let $\mu_{F}$ be the moment map to $\mathbf{f}$ (which we identify with $\mathbf{f}^{*}$ ). Then for $a=n+t$ as above, we have an isomorphism of symplectic manifolds:

$$
X / / a G \approx \mu_{F}^{-1}(t) / F=\left(\mu^{-1}\left(n+\mathrm{f}_{0}\right) / D\right) / /{ }_{t} F .
$$

Since the symplectic form on the right varies linearly with $t$ (which parametrizes $a=n+t)$, we conclude that $\left[\sigma_{a}\right]$ varies linearly with $a$, as claimed.

In a sense, there is linearity even as we degenerate from one stratum to another. We illustrate this phenomenon with the following example.

The left action of $G$ on itself lifts to a Hamiltonian action of $G$ on $X:=$ $T^{*} G \approx G \times \mathfrak{g}^{*}$. The quotient $\operatorname{map} X \rightarrow X / G$ is

$$
\begin{aligned}
G \times \mathfrak{g}^{*} & \rightarrow \mathfrak{g}^{*} \\
(g, a) & \mapsto a,
\end{aligned}
$$

while the moment map sends

$$
(g, a) \mapsto \operatorname{ad}_{g}^{*} a
$$

The symplectic quotients

$$
X / /{ }_{a} G=\mathcal{O}_{a}
$$

are the coadjoint orbits. These fit, as discussed above, into a finite number of continuous families, each indexed by the $W$-quotient of some open face. Inside each family we expect linearity. As we move between families, the cohomology (and even the dimension) jumps. Yet there is a way to express linearity uniformly for all $a \in \mathfrak{g}^{*}$. Let $G_{a}, \mathfrak{g}_{a}$ be the stabilizers of $a$ in $G, \mathfrak{g}$ respectively. Then $a$ restricts to $\bar{a} \in \mathfrak{g}_{a}^{*}$ which is $G_{a}$-invariant, so it gives an element of $\left(\mathfrak{g}_{a}^{*}\right)^{G_{a}}$, a bi-invariant 1 -formon $G_{a}$. This gives a class in $H^{1}\left(G_{a}, \mathbb{C}\right)$, which is then sent to $H^{2}\left(\mathcal{O}_{a}, \mathbb{C}\right)$ by the transgression map (= the coboundary map $E_{2}^{01} \rightarrow E_{2}^{20}$ 
in the Leray spectral sequence of the fibration $G \rightarrow \mathcal{O}_{a}$, with fiber $G_{a}$, where $\left.E_{2}^{p q}=H^{p}\left(\mathcal{O}_{a}, H^{q}\left(G_{a}, \mathbb{C}\right)\right)\right)$. The image is seen to be $\left[\sigma_{a}\right]$, and the construction is obviously linear on each $\mathbf{f}_{0} \subset \mathbf{f} \subset \mathbf{t}$, since the stabilizers are actually constant there.

\section{Which integrable system}

We have seen that all the structures on the quantum moduli space of $N=2$ SYM predicted by Seiberg and Witten can be realized in terms of algebraically integrable systems. The features of "special geometry" occur in any algebraically integrable system. The distinguished electric and magnetic charges appear as soon as we fix a Seiberg-Witten differential, and perhaps the most natural way to get this is as tautological 1-form on a family of curves contained in the total space of a line bundle $\omega_{E}(D)$ of meromorphic differentials on some fixed curve $E$. Since a quantum theory with $\beta=0$ depends on a parameter $\tau=\tau_{c l}$, it is natural to take $E$ to be the elliptic curve corresponding to this $\tau$. Finally, the linearity properties suggest that our family of systems, parametrized by the masses, could arise as the family of symplectic reductions of a larger symplectic manifold.

There remains the technicality of identifying a specific integrable system for each type of theory, and testing that these theories have reasonable physical properties. In this section we first give several descriptions of the system [DW] which solves the $\mathrm{SU}(n)$ theory with one adjoint, and then describe some of the tests which confirm the choice.

\subsection{Meromorphic Higgs bundles}

Fix a curve $E$, an effective divisor $D$ on it, and an integer $n \geq 2$. The total space of the system is the moduli space $X$ of equivalence classes of pairs $(V, \varphi)$, where $V$ is a rank- $n$ vector bundle on $E$ with trivial determinant, $\varphi: V \rightarrow V \otimes \omega_{E}(D)$ is a traceless endomorphism with values in $\omega_{E}(D)$, and the pairs are subject to an appropriate semistability condition. The base is

$$
B:=\oplus_{i=2}^{n} H^{0}\left(E,\left(\omega_{E}(D)\right)^{\otimes i}\right),
$$

and the map $\pi: X \rightarrow B$ sends a pair $(V, \varphi)$ to (the sequence of coefficients of) the characteristic polynomial

$$
\operatorname{det}(t \cdot I-\varphi)
$$

(It is best to think of $t$ as a fiber coordinate taking values in $\omega_{E}(D)$.)

The generic fibers of $\pi$ are abelian varieties. Consider the surface $S$ which is the total space of $\omega_{E}(D)$. The points $b=\left(b_{2}, \ldots, b_{n}\right) \in B$ parametrize a linear system of curves $C_{b} \subset S$, given by the equation $t^{n}+b_{2} t^{n-2}+\cdots+b_{n}=0$. Let $\lambda$ 
be the tautological meromorphic 1 -form on $S$. Then the pair $(V, \varphi)$ determines a sheaf $L:=\operatorname{coker}(\varphi-\lambda \cdot I d)$ on $S$ whose support is the curve $C_{b}$. In fact, for $(V, \varphi)$ generic, $L$ is a line bundle on $C_{b}$. Thus we have a map $\pi^{-1}(b) \rightarrow \operatorname{Pic}\left(C_{b}\right)$. If we were working with $\mathrm{GL}(n)$-bundles rather than $\mathrm{SL}(n)$-bundles (remove the restrictions $\operatorname{det} V \approx \mathcal{O}, \operatorname{Tr}(\varphi)=0$ ), this would be an isomorphism of $\pi^{-1}(b)$ with a component of $\operatorname{Pic}\left(C_{b}\right)$. The restrictions mean that we get instead a translate of $\operatorname{Prym}\left(C_{b} / E\right)$.

This construction, modelled on Hitchin's system [Hi], works much more generally, cf. [D2]. In particular, the structure group $\mathrm{SL}(n)$ can be replaced by any reductive $G$. The total space $X$ now parametrizes pairs $(V, \varphi)$ with $V$ a $G$-bundle over $E$, and $\varphi$ a section of $\operatorname{ad}(V) \otimes \omega_{E}(D)$. The base is replaced by

$$
B:=\oplus_{i=1}^{r} H^{0}\left(E,\left(\omega_{E}(D)\right)^{\otimes d_{i}}\right),
$$

where $r$ is the rank of $G$ and $d_{i}$ are the degrees of the basic invariant polynomials on the Lie algebra $\mathfrak{g}$. Once we choose a representation $\rho$ of $G$, we get a family of spectral curves $\left\{C_{\rho, b} \mid b \in B\right\}$ in $S$ as before.

As shown in [D2], [Fa] and further references therein, the generic fiber of $\pi$ can be identified as (a translate of) a certain distinguished abelian variety $\operatorname{Prym}\left(C_{b}\right)$ which is an isogeny summand in the Jacobian $J\left(C_{\rho, b}\right)$ for each representation $\rho$. The most natural way to view this is to abandon the spectral curves $C_{\rho, b}$ altogether, in favor of the cameral cover $C_{b} \rightarrow E$, a Galois cover with group $W$, the Weyl group of $G$. This is the pullback to $E$, via the classifying map $E \rightarrow \mathbf{t} / W \approx \mathfrak{g} / G$ determined locally in $E$ by the point $b \in B$, of the $W$-cover $\mathbf{t} \rightarrow \mathbf{t} / W$. ( $\mathbf{t}$ is the Cartan subalgebra, and the classifying map is really a map from the total space of $\left(\omega_{E}(D)\right)^{*}$ to $\mathbf{t} / W$. The local form above depends on the choice of a local section of the line bundle $\left(\omega_{E}(D)\right)^{*}$, so is really defined only modulo homotheties). Now $J\left(C_{b}\right)$ decomposes according to the irreducible representations of the Galois group $W$, and the distinguished Prym, $\operatorname{Prym}\left(C_{b}\right)$, is the component corresponding to the action of $W$ on the weights. (The question of the dependence of the spectral Jacobian on $\rho$ was raised in $[\mathrm{AM}]$ and studied, in the case of the Toda system, in [Ml]. A solution, under some conditions, was given in [Ka]. The case $E=\mathbb{P}^{1}$ was analyzed in $[\mathrm{AHH}]$ and $[\mathrm{B}]$, and the general case is in [D2], [Me]). In this section we will use only the $\mathrm{SL}(n)$ system, postponing the appearance of other groups to $\S 4$.

The total space $X$ is not symplectic, but has a Poisson structure [B], [Mn]. In general, a Poisson structure on an algebraic manifold $X$ is a Lie bracket operation on $\mathcal{O}_{X}$ satisfying the properties of the usual Poisson bracket. Equivalently, it can be given by a section $\alpha$ of $\wedge^{2} T_{X}$ satisfying an integrability condition. If $\alpha$ is non-degenerate on some open set $X_{0}$, then its inverse $\sigma=\alpha^{-1}$ is a two-form on $X$, and the integrability condition says that $\sigma$ is closed, i.e. $\sigma$ then gives a symplectic structure. In general, a Poisson manifold admits a natural foliation by submanifolds (whose conormal bundles consist of the null spaces of $\alpha$ ) which inherit a symplectic structure. A typical example is $\mathfrak{g}^{*}$, which has the natural (Kirillov-Kostant) Poisson bracket, the symplectic leaves being precisely the coadjoint orbits. More generally, a Hamiltonian $G$-action on a symplectic man- 
ifold $Y$ induces a Poisson structue on $X=Y / G$. This is the ordinary quotient; the symplectic quotients $X_{a}:=Y / /{ }_{a} G$, for $a \in \mathfrak{g}^{*}$, are recovered as the symplectic leaves of $X$. (The Kirillov-Kostant Poisson structure on $\mathfrak{g}^{*}$ is recovered when we take $\left.Y:=T^{*} G\right)$.

Markman's construction of the Poisson structure is based on the moduli space $\mathcal{M}_{D}$ of "bundles with a level- $D$ structure" on $E: \mathcal{M}_{D}$ parametrizes equivalence classes of stable pairs $(V, \eta)$ where

$$
\eta: V_{D} \stackrel{\sim}{\rightarrow} \longrightarrow \mathcal{O}_{D}^{n}
$$

is a trivialization of the fibers of $V$ at points of $D$ (sending the given volume element of $V_{D}$ to the standard one on $\mathcal{O}_{D}^{n}$, since our structure group is $\left.\mathrm{SL}(n)\right)$. Consider the cotangent bundle $Y:=T^{*} \mathcal{M}_{D}$. By elementary deformation theory, its points are (equivalence classes of) triples $(V, \eta, \varphi)$ with $(V, \eta) \in \mathcal{M}_{D}$ and $(V, \varphi) \in X$, a meromorphic Higgs bundle with poles on $D$. The symmetry group $G$ is the product of copies of $\operatorname{SL}(n)$, one for each point of $D$. (More precisely, $G=\left\{g \in \operatorname{Aut}_{D}\left(\mathcal{O}_{D}^{n}\right) \mid \operatorname{det} g=1\right\}$, allowing for possibly non-reduced $D$.) The $G$ action on $\mathcal{M}_{D}$ (with quotient $\mathcal{M}=\mathcal{M}_{0}$, the moduli of stable bundles on $E$ ) lifts to a Hamiltonian action on $Y$ :

$$
g:(V, \eta, \varphi) \mapsto(V, g \circ \eta, \varphi)
$$

with quotient $X=Y / G$, so we get a Poisson structure on $X$. The moment map

$$
\mu: Y \rightarrow \mathfrak{g}^{*} \approx \mathfrak{g}
$$

sends $(V, \eta, \varphi)$ to $\varphi^{\eta}$, the element of $\operatorname{End}_{D}\left(\mathcal{O}_{D}^{n}\right)$ which is conjugate via $\eta$ to the residue $\operatorname{Res}_{D} \varphi \in \operatorname{End}_{D}\left(V_{D}\right)$. The symplectic leaf $X_{a}$, for $a \in \mathfrak{g}$, can therefore be identified as the moduli space of pairs $(V, \varphi) \in X$ such that $\operatorname{Res}_{D} \varphi$ is conjugate to the given $a$. In particular, each abelian variety $\pi^{-1}(b) \approx \operatorname{Prym}\left(C_{b} / E\right)$ is contained in one symplectic leaf $X_{a}$, and is a Lagrangian subvariety there.

A number of special cases of this construction are well known. When $D=$ 0 , we get Hitchin's system [Hi] on $T^{*} \mathcal{M}$. When $E$ has genus 0 , we get the polynomial matrices system studied in $[\mathrm{AHH}]$ and $[\mathrm{B}]$. For more details and examples, see [Mn] or [DM1].

For the SYM theory with adjoint matter, we take $E$ to be the elliptic curve whose $\tau$ parameter (in the halfplane $\mathbb{H}$ ) is the coupling constant of the theory. We are looking for a 1-parameter family of integrable systems (parametrized by mass $m$ ), each having an $(n-1)$-dimensional base and $(n-1)$-dimensional fibers. One checks easily that $\operatorname{dim}\left(X_{a}\right)$ equals the dimension of the adjoint orbit of $a$; so we are looking for orbits of the smallest possible dimension $2(n-1)$. There is essentially only one 1-parameter family of possibilities: $D$ consists of a single point $\infty \in E$, and $a$ is the conjugacy class of the diagonal matrix with eigenvalues $m(1,1, \ldots, 1,1-n)$. This conjugacy class consists of all diagonalizable $\mathrm{SL}(n)$ matrices $A$ with

$$
\operatorname{rank}(A-m \cdot I d) \leq 1
$$


(As $m \rightarrow 0$ there is one further, nilpotent, orbit of the right dimension: the conjugacy class of the elementary matrix $e_{12}$. But this turns out to give a trivial integrable system, i.e. the same as for $a=0$ : all the spectral curves are completely reducible).

Our system can be described rather explicitly. The moduli space of degree 0 , rank $n$ vector bundles on $E$ can be identified with the symmetric product $S^{n} E$ : each equivalence class contains a unique decomposable bundle $V=\oplus_{i=1}^{n} L_{i}$, with $L_{i}=\mathcal{O}_{E}\left(q_{i}-\infty\right) \in \operatorname{Pic}^{0} E$. The moduli of $\operatorname{SL}(n)$-bundles is the fiber over 0 of the Abel-Jacobi map $S^{n} E \rightarrow E$. It is a projective space

$$
\left\{q_{1}+\cdots+q_{n} \mid \sum q_{i}=n \infty\right\}=\mathbb{P} H^{0}(E, \mathcal{O}(n \infty)) \approx \mathbb{P}^{n-1} .
$$

In terms of the decomposition $V=\oplus L_{i}$, any Higgs field $\varphi$ on $V$ can be written as a matrix with entries

$$
\varphi_{i j} \in \Gamma\left(L_{j} \otimes L_{i}^{-1}(\infty)\right) .
$$

Let $A:=\operatorname{Res}_{\infty} \varphi$. Its diagonal elements are all zeroes, and $A-m \cdot I d$ has rank $\leq 1$, so $A$ is conjugate, via a diagonal matrix, to $m$ times $e=\sum_{i \neq j} e_{i j}$. This means that each $(V, \varphi)$ in our system is equivalent to a pair $(V, \varphi)$ with $\operatorname{Res}_{\infty}\left(\varphi_{i j}\right)=m$ for $i \neq j$. For a given $V$, this condition uniquely determines the $\varphi_{i j}$ (as long as $L_{i} \neq L_{i}$ ). The remaining free parameters are the coordinates $q_{i}$ of $V=\oplus \mathcal{O}\left(q_{i}-\infty\right)$ and the diagonal entries $p_{i}=\varphi_{i i}$. A way from the diagonals $q_{i}=q_{j}$, these $\left(q_{i}, p_{i}\right)$ can be identified with the canonical coordinates on $T^{*} \mathcal{M}$.

\subsection{The spectral curves}

The family of spectral curves $C_{b}$, for the fundamental, $n$-dimensional representation of $\mathrm{SL}(n)$, can be described as a linear system on the compactification $\bar{S}=\mathbb{P}\left(\omega_{E}(\infty) \oplus \mathcal{O}_{E}\right)$ of the surface $S$, or on various birationally equivalent surfaces. More precisely, it forms an affine open subspace of this linear system, the complement consisting of curves which contain the section at infinity, $Z_{\infty}:=\bar{S}-S$, with some multiplicity $k>0$. By removing this multiple component, we recover the spectral curves for the analogous problem with $n$ replaced by $n-k$. (If $A$ is an endomorphism of a vector space $V$, and $W$ is an invariant subspace, then

$$
\operatorname{rank}_{W}(A-m \cdot I d) \leq \operatorname{rank}_{V}(A-m \cdot I d),
$$

so the conjugacy class restricts correctly.)

We can write down an explicit equation

$$
p_{n}(t)=t^{n}+\sum_{k=2}^{n} f_{k} t^{n-k}=0
$$

for the spectral curves. Here $t$ is a coordinate on the fibers of $\omega_{E}(\infty)$, and $f_{k} \in \Gamma\left(\mathcal{O}_{E}(k \infty)\right)$. (We identify $\left.\mathcal{O}_{E} \approx \omega_{E}\right)$. Once we have such a $p_{n}$ for each 
$n$, the previous remark and the tracelessness condition imply that the general spectral curve has equation

$$
C_{u}: p_{n}+\sum_{\ell=0}^{n-2} u_{\ell} p_{\ell}=0,
$$

with arbitrary constants $u_{\ell}$ which can therefore be interpreted as coordinates on the quantum moduli-space, the base of the integrable system.

The condition on $p_{n}$ which specifies the right conjugacy class at infinity is that when we substitute $t=t^{\prime}+\xi^{-1}$, where $\xi$ is a local coordinate on $E$ at $\infty$, we get a function of $t^{\prime}$ and $\xi$ with at most first order poles in $\xi$. Write the equation of $E$ in Weierstrass form,

$$
E: y^{2}=x^{3}+b x-c .
$$

The local coordinate $\xi$ determines a sequence $x_{k}, k=2,3, \ldots$, of rational functions on $E$ which are regular on $E-\infty$ and such that near $\infty$,

$$
x_{k}-\xi^{-k}=O\left(\xi^{-1}\right) \text {. }
$$

We may as well use the local coordinate $\xi=x^{-1 / 2}$, and then the $x_{k}$ can be read off the Taylor expansions:

$y=\xi^{-3}\left(1+b \xi^{4}-c \xi^{6}\right)^{1 / 2}=\xi^{-3}\left(1+\frac{b}{2} \xi^{4}-\frac{c}{2} \xi^{6}-\frac{b^{2}}{8} \xi^{8}+\frac{b c}{4} \xi^{10}-\frac{2 c^{2}-b^{3}}{16} \xi^{12}+\ldots\right)$

and

$$
\begin{aligned}
y^{-1}=\xi^{3}\left(1+b \xi^{4}-c \xi^{6}\right)^{-1 / 2} & \\
& =\xi^{3}\left(1-\frac{b}{2} \xi^{4}+\frac{c}{2} \xi^{6}+\frac{3 b^{2}}{8} \xi^{8}-\frac{3 b c}{4} \xi^{10}+\frac{6 c^{2}-5 b^{3}}{16} \xi^{12}+\ldots\right) .
\end{aligned}
$$

Explicitly, we get:

$$
\begin{aligned}
x_{2} & =x \\
x_{3} & =y \\
x_{4} & =x^{2} \\
x_{5} & =x y \\
x_{6} & =x^{3} \\
x_{7} & =x^{2} y-\frac{b}{2} y \\
x_{8} & =x^{4} \\
x_{9} & =x^{3} y-\frac{b}{2} x y+\frac{c}{2} y \\
x_{10} & =x^{5} \\
x_{11} & =x^{4} y-\frac{b}{2} x^{2} y+\frac{c}{2} x y+\frac{3 b^{2}}{8} y \\
x_{12} & =x^{6},
\end{aligned}
$$


and so on. In terms of this basis, one checks by an easy induction argument that

$$
p_{n}=t^{n}-\sum_{k=2}^{n}(-1)^{k}(k-1)\left(\begin{array}{l}
n \\
k
\end{array}\right) x_{k} t^{n-k},
$$

so the first few polynomials are:

$$
\begin{aligned}
& 1 \\
& t \\
& t^{2}-x \\
& t^{3}-3 x t+2 y \\
& t^{4}-6 x t^{2}+8 y t-3 x^{2} \\
& t^{5}-10 x t^{3}+20 y t^{2}-15 x^{2} t+4 x y \\
& t^{6}-15 x t^{4}+40 y t^{3}-45 x^{2} t^{2}+24 x y t-5 x^{3} \\
& t^{7}-21 x t^{5}+70 y t^{4}-105 x^{2} t^{3}+84 x y t^{2}-35 x^{3} t+6\left(x^{2} y-\frac{b}{2} y\right) .
\end{aligned}
$$

This is a bit cleaner than the formulas in [DW], mostly because we set the sum of the roots in the Weierstrass equation of $E$ to 0 . A more-or-less equivalent solution was given already in [T], where the generating function for the $p_{n}$ was written in terms of theta functions and exponentials on $E$. A slightly different (but equivalent) set of polynomials was given in [IM1].

\subsection{Elliptic solitons}

It is curious to note that the same system arises in several rather different contexts. Here is a brief outline of one of these, following the work of Treibich and Verdier [T], [TV].

Finite dimensional solutions of the KP hierarchy (= infinite dimensional integrable system) are well understood. They are parametrized by Krichever data, consisting of a curve $C$, a point $p \in C$, a line bundle $L$, and local trivializations. Such data determine a point in the total space of the hierarchy with the property that the infinite collection of commuting KP flows emanating from the point sweep out only a finite dimensional space, isomorphic to $J(C)$. The curve $C$, the point $p$ and the trivializations remain fixed, while $L$ moves freely in $J(C)$.

An elliptic soliton is a finite dimensional solution of KP in which the first flow, $K P_{1}$, already closes up, i.e. evolves on an elliptic curve inside the possibly larger Jacobian $J(C)$. The direction of $K P_{1}$, for points coming from Krichever data, is the tangent vector at $p$ to the Abel-Jacobi image of $C$ in $J(C)$. So the condition is that $J(C)$ should contain an elliptic curve $E$ which is tangent at $A J(p)$ to the Abel-Jacobi image $A J(C)$.

Start with an $n$-sheeted branched cover $\pi: C \rightarrow E$ and a point $p \in E$ with local coordinate $\xi$. Let $\pi^{*}(p)=q_{0}+\cdots+q_{n-1}$, and let $\xi_{i}:=\xi \circ \pi$ near $q_{i}$. The 
tangent vector $t_{i}:=\partial / \partial \xi_{i}$ lives naturally in $\Gamma\left(\mathcal{O}_{q_{i}}\left(q_{i}\right)\right)$. Its image

$$
(A J)_{*} t_{i} \in T_{A J\left(q_{i}\right)} J(C)
$$

can then be identified with the image of $t_{i}$ under the coboundary map $\delta$ for the sequence

$$
0 \rightarrow \mathcal{O}_{C} \rightarrow \mathcal{O}_{C}\left(q_{i}\right) \rightarrow \mathcal{O}_{q_{i}}\left(q_{i}\right) \rightarrow 0
$$

Similarly, the tangent at $\pi^{*} p$ to the image $\pi^{*} E$ of $E$ in $J(C)$ is given by $\sum_{i=0}^{n-1} \delta\left(t_{i}\right)=\delta\left(\sum_{i=0}^{n-1} t_{i}\right)$. The tangency condition then says that $\delta\left(\sum_{i=0}^{n-1} t_{i}\right)$ is proportional to $\delta\left(t_{0}\right)$, and therefore equal (by the residue theorem) to $\delta\left(n t_{0}\right)$. This happens if and only if there is some $f \in \Gamma\left(\mathcal{O}\left(\pi^{*} p\right)\right)$ with

$$
\operatorname{Res}_{q_{i}}\left(f \pi^{*} d z\right)= \begin{cases}1, & 1 \leq i \leq n-1 \\ 1-n, & i=0\end{cases}
$$

where $d z$ is a non-zero closed 1-form on $E$. This is equivalent to saying that $\pi: C \rightarrow E$ is one of our spectral curves, and $f \pi^{*} d z$ becomes the Seiberg-Witten differential.

\subsection{Tests}

What makes this description of SYM with adjoint matter convincing for a physicist, is that it can be "tested" by working out various limits and special cases, and that it yields results consistent with the "predictions" based on the expected behavior of the physical system. We outline some of these tests.

\subsection{Consistency with Seiberg-Witten}

For $n=2$, with given $E$ and $m$, our system produces a 1-parameter family of double covers $C_{u} \rightarrow E$. Each $C_{u}$ has genus 2, and the integrable system is the family of 1-dimensional Pryms. Write the equation of $E$ as $y^{2}=\prod_{i=1}^{3}\left(x-e_{i}\right)=$ $x^{3}+b x-c$, with $\sum e_{i}=0$ as before. The cover $C_{u}$ is given explicitly in terms of our polynomials $p_{i}$ as:

$$
0=p_{2}+u p_{0}=t^{2}-x+u
$$

Its Galois group over the $\mathbb{P}^{1}$ with coordinate $x$ is $\mathbb{Z}_{2} \times \mathbb{Z}_{2}$, with involutions $\alpha: y \mapsto-y,, \beta: t \mapsto-t$, and $\alpha \beta$. The quotients are a rational curve (with coordinate $t$ ); the original $E$; and another elliptic curve $E_{u}$, respectively. 


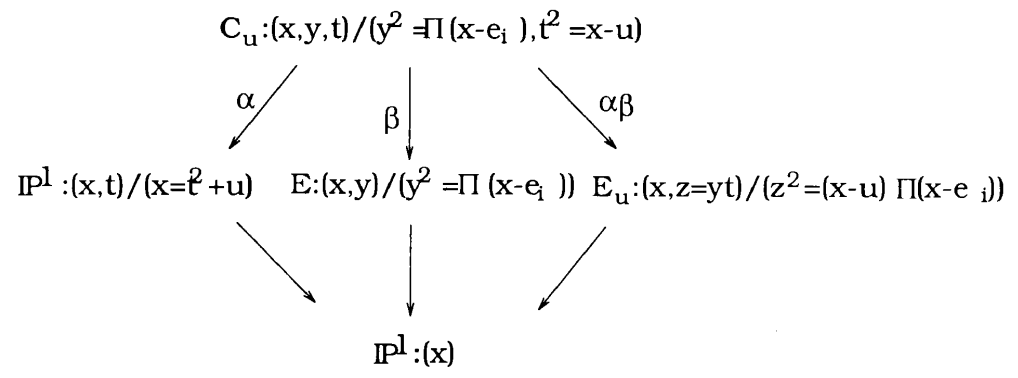

The Prym can then be identified with $E_{u}$.

The original Seiberg-Witten solution is given directly as the family of elliptic curves $E_{u}^{\prime}$, double covers of $\mathbb{P}^{1}$ branched at $e_{i} u+e_{i}^{2} \quad(i=1,2,3)$ and $\infty$ ([SW2], eq. (16.17)). These are indeed isomorphic to our $E_{u}$ : the transformation

$$
x \mapsto \frac{\left(u^{2}+b\right) x-c}{u-x}
$$

takes the $e_{i}$ and $u$ to $e_{i} u+e_{i}^{2}$ and $\infty$, respectively.

\subsection{Mass to zero: the $N=4$ limit}

The limit $m \rightarrow 0$ of $N=2$ SYM with gauge group $G$ and adjoint matter is known to acquire a higher form of supersymmetry: it is the $N=4 \mathrm{SYM}$ theory, with the same gauge group. (A physicist might rather say that two of the four supersymmetries of $N=4 \mathrm{SYM}$ are broken when the theory is perturbed by the addition of a mass term to the Lagrangian). The extra symmetry of the $N=4$ theory means that "everything" (i.e. the ingredients of the special geometry package encountered in $\S 2$ : the quantum moduli space, the Kähler metric, the prepotential...) can be computed explicitly. The outcome matches perfectly to the corresponding quantities for Hitchin's system [Hi], i.e. the system of holomorphic (no poles) Higgs bundles on $E$. (This computation is in $\S 2.3$ of [DW]). So the physics predicts that our system should specialize, as $m \rightarrow 0$, to Hitchin's for $\mathrm{SU}(n)$, which it of course does. (This is not really an honest "test", since we started out searching for deformations of Hitchin's system, so this limit was built in from the beginning).

\section{7 $\tau$ to $\infty$ : the flow to pure $N=2$}

One of the most interesting limits occurs as both $m$ and $\tau$ go to infinity simultaneously, with $\tau$ proportional to $\log (m)$. Equivalently, after an obvious rescaling, we can keep $m=1$ and let the elliptic curve degenerate $(\tau \rightarrow \infty)$ while adjusting the coefficients $u_{\ell}$ in the spectral curves $C_{u}$.

The physics of this limit is easy to work out. Essentially, the mass going to infinity means that in this limit the particle is so heavy that it cannot be affected by the rest of the system; we get the pure $\mathrm{N}=2$ theory, with no mass. 
This theory was solved in [AF] and [KLTY]. The solution does not involve $\tau$, as we saw in the section on adding matter: instead of $\tau$ having a limit (which was there called $\tau_{c I}$ ), it now goes to $\infty$. The spectral curves found in [AF] and [KLTY] have the explicit equation:

$$
C_{b}^{\prime}: w^{2}=\left(z^{n}+b_{2} z^{n-2}+\cdots+b_{n}\right)^{2}+1
$$

in the $(z, w)$-plane, where the coefficients $b_{k}$ play a role analogous to our $u_{\ell}$.

In [DW], we show that these curves are indeed recovered as the appropriate limits of the spectral curves for the theory with adjoint mass. This is done by substituting the $\tau \rightarrow \infty$ limit into the explicit equations obtained above for the $C_{u}$, and performing the relevant rescalings. Instead of repeating the derivation here, we will describe the geometry of this degeneration.

The pure $\mathrm{N}=2$ curve $C_{b}^{\prime}$ is the fibre product over $\mathbb{P}^{1}$ (with coordinate $v$ ) of the rational two-sheeted cover $w^{2}=v^{2}+1$ and the (again rational, with coordinate $z$ ) n-sheeted cover $v=z^{n}+b_{2} z^{n-2}+\cdots+b_{n}$. In the adjoint theory, the analogue of the double cover is just the elliptic curve $E \rightarrow \mathbb{P}^{1}$ (which becomes rational in the limit $\tau \rightarrow \infty$ ), and $C_{u}$ is again an n-sheeted branched cover of $E$, but it is no longer the fiber product of $E$ with any $n$-sheeted cover of $\mathbb{P}^{1}$. The degree 2 map $\psi: C \rightarrow \mathbb{P}_{z}^{1}$ and the degree $n$ map $\mathbb{P}_{z}^{1} \rightarrow \mathbb{P}_{v}^{1}$ arise only in the limit: $C$ becomes hyperelliptic.

The type of corank-1 degeneration involved in going from $C_{u}$ to $C_{b}^{\prime}$ can be seen more generally. Consider a degree $n$ branched cover $\pi: C \rightarrow E$ where $E$ is elliptic and $C$ has genus $\mathrm{n}$. Up to isogeny, the Jacobian decomposes $J(C) \approx P \times E$, where $P$ is the Prym. There are the Abel-Jacobi and AbelPrym maps:

$$
\begin{gathered}
A J: C \rightarrow J(C) \\
A P: C \rightarrow P
\end{gathered}
$$

and their derivatives

$$
\begin{gathered}
\phi: C \rightarrow \mathbb{P}^{n-1} \\
\psi: C \rightarrow \mathbb{P}^{n-2} .
\end{gathered}
$$

Here $\phi$ is the canonical map and $\psi$ is the composition of $\phi$ with linear projection from the point $0 \in \mathbb{P}^{n-1}$ determined by the isogeny.

In the degeneration, we want each of $E, C$ to acquire a node: the singular curves are $E_{0}, C_{0}$, with normalizations $E^{\prime}, C^{\prime}$, and singular points $p_{0} \in$ $E_{0}, q_{0} \in C_{0}$ with branches $p_{i}, q_{i}, i=1,2$ in the normalizations. The Jacobians $J(C), J(E)$ become non-compact: $J\left(E_{0}\right)$ is a $C^{*}$, while $J\left(C_{0}\right)$ is a $C^{*}$-extension of $J\left(C^{\prime}\right)$; but the Prym $P$ remains compact. This type of degeneration occurs when $\pi^{\prime}: C^{\prime} \rightarrow E^{\prime}$ is totally ramified above each of the $p_{i}: \pi^{\prime-1}\left(p_{i}\right)=n q_{i}$. In this case we still have the Abel-Jacobi and Abel-Prym maps, going to the compactified Jacobians. The derivative $\phi$ is still the canonical map of the singular $C_{0}$; its image has a node at $0=\phi\left(q_{0}\right)$. On the other hand, $\psi$ is no longer a morphism on $C_{0}$; its restriction $\psi^{\prime}: C^{\prime} \rightarrow \mathbb{P}^{n-2}$ is now the canonical map of $C^{\prime}$. 
Within the $2 n-2$-dimensional family of all covers $\pi: C \rightarrow E$ we restrict attention to the $n$-dimensional family of our spectral curves. Here there exist points $p \in E, q \in C$ such that $A J(C)$ at $q$ is tangent to $\pi^{*}(E)$ at $p$. This is equivalent to saying that $A P: C \rightarrow P(C / E)$ is ramified at $q$, or that the linear system giving $\psi$ has a base point at $q$, or that $\phi(q)=0$.

Combining these two effects, we find that when the corank- 1 degeneration described above occurs within the family of spectral curves, the two branches $\phi\left(q_{1}\right), \phi\left(q_{2}\right)$ are tangent at 0 . Equivalently, the canonical map $\psi^{\prime}$ satisfies $\psi\left(q_{1}\right)=\psi\left(q_{2}\right)$; so $C^{\prime}$ is hyperelliptic, $\psi$ is two-to-one, and $\mathcal{O}\left(p_{1}+p_{2}\right)$ is the hyperelliptic bundle. It follows immediately that the two maps, of degrees 2 and $n$ respectively, from $C^{\prime}$ to $\mathbb{P}^{1}$ fit in a cartesian diagram. The corank-1 degeneration of the spectral curves (for the adjoint theory) therefore reproduces exactly the spectral curves of the pure $N=2$ theory.

\subsection{Higgs to $\infty$ : symmetry breaking}

This time we fix both the curve $E$ and the mass $m$, and instead we flow to the theory whose gauge group is some Levi subgroup $G \subset \mathrm{SU}(n)$. Fix a diagonal matrix $\psi$, and consider the flow sending a Higgs bundle $(V, \varphi)$ in our system to $\left(V, \varphi_{s}\right)$ where $\varphi_{s}:=\varphi+s \psi$. (As before we decompose $V=\oplus L_{i}$ and let $\psi$ act diagonally. The flow is well-defined only on the $n$ !-sheeted cover of the system on which this decomposition exists). The physics predicts that the limit of the $\mathrm{SU}(n)$ integrable system as $s \rightarrow \infty$ along this flow should be the $G$-system, where $G \subset \mathrm{SU}(n)$ is the centralizer of $\psi$.

For generic $\psi$, with distinct eigenvalues, this is clear: After rescaling we have $\varphi_{s} \sim \varphi_{s}^{\prime}:=\psi+\frac{1}{s} \varphi$, so the limiting spectral curve is $\operatorname{det}(t-\psi)=0$, which consists of $n$ copies of $E$ meeting at $\infty$. The Prym is then the product $J(E)^{(n-1)}$, independent of the initial $(V, \varphi)$. This trivial system corresponds to the Abelian structure group $\mathrm{U}(1)^{n-1}$ which is the maximal torus of $\mathrm{SU}(n)$ and the centralizer of $\psi$.

In general, $\psi$ will have, say, $k$ distinct eigenvalues with multiplicities $n_{1}, \ldots, n_{k}, \sum n_{i}=n$. The centralizer is

$$
G=\mathrm{U}(1)^{k-1} \times \prod_{i=1}^{k} \mathrm{SU}\left(n_{i}\right) .
$$

Now the limit of the spectral curves of $\varphi_{s}^{\prime}$ is non-reduced, consisting of $k$ components, the $i$ th with multiplicity $n_{i}$. What we need is the limit of the Jacobians. This clearly has a factor (up to isogeny) from each component, and by rescaling around the $i$ th eigenvalue $\psi_{i}$ of $\psi$ (i.e. setting $t-\psi_{i}=s^{-1} \tilde{t}$ ) we see that this factor is the Jacobian of the spectral curve

$$
\operatorname{det}\left(\tilde{t}-\varphi_{i}\right)
$$

where $\varphi_{i}$ is the $n_{i} \times n_{i}$ dimensional $i$ th block of $\varphi$. The claim is then that this is one of the $\mathrm{SU}\left(n_{i}\right)$ spectral curves, i.e. that the residue at $\infty$ of the $i$ th 
block is conjugate to $m \cdot \operatorname{diag}\left(1,1, \ldots, 1,1-n_{i}\right)$. An equivalent condition is: $\operatorname{rank}\left(\varphi_{i}-m\right) \leq 1$, and this condition is clearly inherited by any block in $\varphi$, as required.

\subsection{Singularities}

Much of the effort in [DW] was devoted to analyzing the discriminant, the hypersurface $D \subset B$ over which the spectral curves are singular. Physically, that is where all the interesting phenomena occur, and where many predictions can be made. For instance, as $\tau \rightarrow \infty$ ("at weak coupling"), the limit of $D$ should be reducible: this is a classical limit, where the mass spectrum can be analyzed directly from the classical Lagrangian. $D$ is the locus where some mass vanishes. This mass can belong to either of the two types (vector or hyper) of $N=2$ SUSY multiplets mentioned in section 1.8 ("Adding matter"), and this gives rise to the two components. In fact, one knows the explicit equations in $B$ for these components. A Maple assisted computation, using the explicit equations obtained above for the spectral curves, confirmed the reducibility and produced the precise predicted factors for $\mathrm{SU}(3)$.

For any $\tau$ (and any $n$ ), the most interesting locus in $B$ is the $(n-1)$-fold self-intersection of $D$, a finite set. Physically, this corresponds to the massive vacua, where the (low energy) gauge group is broken to its maximal torus by monopole condensation. A classification result due to 't Hooft predicts that the possible massive vacua should correspond to index $n$ subgroups of $\mathbb{Z}_{n} \times \mathbb{Z}_{n}$.

The corresponding result can be obtained directly from the integrable system. The general spectral curve has genus $n$, and we are imposing the maximal number, $n-1$, of nodes. The normalized spectral curve is then of genus 1 , and must be an unramified $n$-sheeted cover of the base $E$. These covers are of course classified by the subgroups as above. Conversely, given any $n$-sheeted cover $\pi: E^{\prime} \rightarrow E$ and a point $\infty^{\prime}$ above $\infty \in E$, there is a unique meromorphic 1 -form on $E^{\prime}$ with residue $m$ at $\pi^{-1}(\infty) \backslash \infty^{\prime}$ and residue $(1-n) m$ at $\infty^{\prime}$ (modulo the constant form $\pi^{*} d z$ ), hence a unique map of $E^{\prime}$ to the total space of $\omega_{E}(\infty)$ whose image is a spectral curve, as required. (Changing the choice of $\infty^{\prime}$ amounts to a deck transformation, leaving the spectral curve unchanged).

We also analyzed, assisted with some massive and miraculous-looking Maple computations, the locus of cusps in the $\mathrm{SU}(3)$ theory, again confirming some rather delicate predictions coming from the physics. It seems that there is some very beautiful geometry still waiting to be understood in the structure of the discriminant for the $\mathrm{SU}(n)$ theories.

\section{Other integrable systems}

Having studied in some detail the system which solves one particular family of SYM theories, we proceed to consider what is known for some of the other families. Our discussion will include, 
- The pure $N=2$ SYM for arbitrary gauge group $G$, and its relation to the Toda systems.

- The situation when fundamental matter is added to the pure theory.

- The theory with adjoint matter for arbitrary $G$, and its relation to the elliptic Calogero-Moser system.

- A few words about the integrable systems arising in string theory.

\subsection{Pure $N=2$ SYM}

The solutions by Seiberg and Witten of the pure $N=2$ SYM theory [SW1] and the theories with fundamental or adjoint matter [SW2] led rapidly to the creation of a whole literature of solutions to specific SYM theories. The general pattern was: postulate the general form of a family of curves which buildsin some of the expected symmetries of the theory, then adjust coefficients to enforce all other available information: further symmetries, limiting theories which were already known, internal consistencies. All cases which were worked out this way, e.g. [KLTY], [AF], [DS], [HO], [APS], [BL], [AS], [H], [AAG] and further references therein, were based on postulating curves which are hyperelliptic or closely related to hyperelliptics.

It was observed in [GKMMM] that the curves obtained in [KLTY], [AF] for the pure $G=\mathrm{SU}(n)$ theory are precisely the spectral curves of the periodic Toda system. The analogue for all semisimple groups was found in [MW], which we follow here.

\subsection{The Toda system}

A simple way to describe the Toda system is via the construction of AdlerKostant-Symes. Start with a decomposition $\mathfrak{g}=\mathbf{a} \oplus \mathbf{b}$ of a Lie algebra $\mathfrak{g}$ as vector space direct sum of two of its subalgebras $\mathbf{a}, \mathbf{b}$. This induces a dual decomposition $\mathfrak{g}^{*}=\mathbf{a}^{*} \oplus \mathbf{b}^{*}$, an injection $i:=\mathbf{b}^{*} \hookrightarrow \mathfrak{g}^{*}$, and a projection $\pi: \mathfrak{g} \rightarrow \mathbf{b}$. Let $\mathcal{O}_{x} \subset \mathbf{b}^{*}$ denote the coadjoint orbit of some $x \in \mathbf{b}^{*}$. The image $i\left(\mathcal{O}_{x}\right) \subset \mathfrak{g}^{*}$ has, in general, nothing to do with the coadjoint orbit $\mathcal{O}_{i(x)}$ of $i(x)$ in $\mathfrak{g}^{*}$. So $i^{*}$ of a $G$-invariant function $f$ on $\mathfrak{g}^{*}$ can be a complicated, non-constant function on $\mathcal{O}_{x}$. The AKS claim is that any two such, $i^{*} f_{1}$ and $i^{*} f_{2}$, Poisson commute with respect to the natural (Kirillov-Kostant) symplectic structure $\sigma_{\mathcal{O}_{x}}$ on $\mathcal{O}_{x}$. Indeed, let $v_{j} \in \mathfrak{g}$ correspond to the differential $\left.d f_{j}\right|_{i(x)} \in \mathfrak{g}^{* *}=\mathfrak{g}$, so $i^{*} d f_{j}$ is given by $\pi\left(v_{j}\right)$. $G$-invariance of $f_{j}$ implies that $v_{j}$ is conormal to $\mathcal{O}_{i(x)}$, i.e. that

$$
0=\left\langle i(x),\left[\mathfrak{g}, v_{j}\right]\right\rangle
$$


We set $a_{j}:=v_{j}-\pi\left(v_{j}\right) \in \mathbf{a}$, and compute:

$$
\begin{aligned}
\left\{i^{*} f_{1}, i^{*} f_{2}\right\}_{\mathcal{O}_{x}} & =\sigma_{\mathcal{O}_{x}}\left(\pi v_{1}, \pi v_{2}\right) \\
& =\left\langle x,\left[\pi\left(v_{1}\right), \pi\left(v_{2}\right)\right]_{\mathbf{b}}\right\rangle \\
& =\left\langle i(x),\left[\pi\left(v_{1}\right), \pi\left(v_{2}\right)\right]_{\mathfrak{g}}\right\rangle \\
& =\left\langle i(x),\left[v_{1}, \pi\left(v_{2}\right)\right]_{\mathfrak{g}}\right\rangle-\left\langle i(x),\left[a_{1}, \pi\left(v_{2}\right)\right]_{\mathfrak{g}}\right\rangle \\
& =\left\langle i(x),\left[v_{1}, \pi\left(v_{2}\right)\right]\right\rangle-\left\langle i(x),\left[a_{1}, v_{2}\right]\right\rangle+\left\langle i(x),\left[a_{1}, a_{2}\right]\right\rangle .
\end{aligned}
$$

We have just seen that the first two terms vanish when $f_{1}, f_{2}$ are $G$-invariant, and the third term vanishes since it pairs $i(x) \in \mathbf{b}^{*}=\mathbf{a}^{\perp}$ with $\left[a_{1}, a_{2}\right] \in \mathbf{a}$. This proves AKS.

A shifted version of AKS is very convenient in applications. Replace the inclusion $i: \mathbf{b}^{*} \hookrightarrow \mathfrak{g}^{*}$ by its shifted version $\epsilon+i$, where $\epsilon$ is an element of $\mathfrak{g}^{*}$ which annihilates $[\mathbf{a}, \mathbf{a}]$ and $[\mathbf{b}, \mathbf{b}]$. The above argument goes through verbatim. For example, take $\mathfrak{g}=\mathbf{s l}(n), \mathbf{a}=$ strictly lower triangular matrices, $\mathbf{b}=$ upper triangulars, and (after identifying $\mathfrak{g}$ with $\mathfrak{g}^{*}$, so that $\mathbf{b}^{*}$ becomes the lower triangulars):

$$
\epsilon=\left(\begin{array}{ccccc}
0 & 1 & & & \\
& 0 & & & \\
& & \ddots & & \\
& & & 0 & 1 \\
& & & & 0
\end{array}\right), x=\left(\begin{array}{ccccc}
0 & & & & \\
1 & 0 & & & \\
& & \ddots & & \\
& & & 0 & \\
& & & 1 & 0
\end{array}\right) .
$$

The orbit $\mathcal{O}_{x} \subset \mathbf{b}^{*}$ consists of all matrices of the form:

$$
\left(\begin{array}{ccc}
b_{1} & & 0 \\
a_{1} & \ddots & \\
& \ddots & \\
0 & & a_{n-1} b_{n}
\end{array}\right)
$$

with $a_{j} \neq 0$ and $\sum b_{j}=0$. The shifted orbit consists of tridiagonal matrices:

$$
\left(\begin{array}{ccccc}
b_{1} & 1 & & & 0 \\
a_{1} & \cdot & \cdot & & \\
& \cdot & \cdot & \cdot & \\
& & \cdot & \cdot & 1 \\
0 & & & a_{n-1} & b_{n}
\end{array}\right) .
$$

This is a $2(n-1)$-dimensional symplectic manifold $(X, \sigma)$, isomorphic as symplectic manifold to the cotangent bundle of the maximal torus $T \approx\left(\mathbb{C}^{*}\right)^{n-1}$. The restriction to it of the symmetric polynomials of $\mathrm{SL}(n)$ (= coefficients of the characteristic polynomial) gives $n-1$ independent, commuting Hamiltonians. This is the classical Toda system. 
An equivalent system is obtained if $\epsilon$ is replaced by $m \cdot \epsilon$ with $m \in \mathbb{C}^{*}$. When $m=0$, the Hamiltonians become the symmetric functions of the $b_{i}$. We get the cotangent bundle $X$ of $\left(\mathbb{C}^{*}\right)^{n-1}$, fibered by its moment map over $(\mathbb{C})^{n-1}$. Another variant is to take $\epsilon=0$, but replace a by the algebra $\operatorname{so}(n)$ of skew symmetric matrices. Now $\mathbf{b}^{*}$ sits in $\mathbf{s l}(n)$ as the symmetric matrices, and the orbit consists of symmetric tridiagonal matrices

$$
\left(\begin{array}{ccccc}
b_{1} & c_{1} & & & \\
c_{1} & b_{2} & & & \\
& & \ddots & & \\
& & & b_{n-1} & c_{n-1} \\
& & & c_{n-1} & b_{n}
\end{array}\right) .
$$

This is conjugate to the previous matrix with $a_{i}=c_{i}^{2}$, so we obtain a new version $(\tilde{X}, \tilde{\sigma})$ of Toda which is just a $2^{n-1}$-sheeted cover of the previous, basic version, $(X, \sigma)$.

The Toda system for a semisimple Lie algebra $\mathfrak{g}$ is obtained with $\mathbf{b}$ a Borel subalgebra, a the nilpotent radical of the opposite Borel, $x=\sum e_{\alpha}$ and $\epsilon=$ $\sum e_{-\alpha}$, where the sums are over the simple roots $\alpha$ with respect to $\mathbf{b}$, and the $e_{ \pm \alpha}$ are normalized root vectors. The total space can again be identified with the cotangent bundle of the maximal torus, a typical element having the form $\sum_{\alpha \text { simple }}\left(b_{\alpha} h_{\alpha}+a_{\alpha} e_{\alpha}+e_{-\alpha}\right)$ with respect to a standard basis $h_{\alpha}, e_{ \pm \alpha}$.

\subsection{Periodic Toda}

The periodic Toda systems are obtained when the semisimple algebra $\mathfrak{g}$ is replaced by a loop algebra $\mathfrak{g}^{(1)}$. This has an extended Dynkin diagram consisting of the simple roots $\alpha$ of $\mathfrak{g}$ plus the affine root $\alpha_{0}$, corresponding to the highest root of $\mathfrak{g}$. The general element of the periodic Toda system can thus be written as

$$
\sum_{\alpha}\left(b_{\alpha} h_{\alpha}+a_{\alpha} e_{\alpha}+e_{-\alpha}\right)+z e_{\alpha_{0}}+z^{-1} a_{0} e_{-\alpha_{0}}
$$

where $z$ is the loop variable, and the product $a_{0} \prod_{\alpha} a_{\alpha}=\mu$ is a fixed non-zero constant. (For $\mathfrak{g}=\mathbf{s l}(n)$, this is:

$$
\left(\begin{array}{cccc}
b_{1} & 1 & & a_{0} z^{-1} \\
a_{1} & \ddots & \ddots & \\
& \ddots & \ddots & 1 \\
z & & a_{n-1} & b_{n}
\end{array}\right),
$$

with $\left.\prod a_{i}=\mu, \sum b_{j}=0\right)$.

As in the case of Higgs bundles, the spectral curves $C_{\rho, b}$ depend on the choice of a representation $\rho$ of $G$. These Toda curves and their relationships were first studied in $[\mathrm{Ml}]$ and $[\mathrm{MS}]$, with some clarifications added in [MW1]. 
Each $C_{\rho, b}$ is a branched cover of the $z$-line $\mathbb{P}_{z}^{1}$. The latter plays the role for periodic Toda which the base curve $E$ played for the meromorphic Higgs system in $\S 2$. The cameral cover in this situation has a strikingly simple description. The classifying map $\mathbb{P}_{z}^{1} \rightarrow \mathbf{t} / W$, which is usually only locally defined, is now a morphism for $z \neq 0, \infty$. It factors through the double cover

$$
\begin{aligned}
\mathbb{P}_{z}^{1} & \rightarrow \mathbb{P}_{w}^{1} \\
z & \mapsto w=z+\mu z^{-1}
\end{aligned}
$$

which maps $\mathbb{P}_{z}^{1} \backslash\{0, \infty\}$ to $\mathbb{A}_{w}^{1}=\mathbb{P}_{w}^{1} \backslash\{\infty\}$, followed by an affine linear map

$$
\mathbb{A}_{w}^{1} \mapsto \mathbf{t} / W
$$

whose image in $\mathbf{t} / W$ is a straight line pointing in the direction of the invariant polynomial of highest degree, which is equal to the Coxeter number $h_{\mathfrak{g}}$ of $\mathfrak{g}$. More precisely, the algebra of polynomial functions on $\mathbf{t} / W$ is isomorphic to the algebra of invariant polynomials on $t$, which is filtered by the degree. The equations of the image of $\mathbb{A}_{w}^{1}$ are obtained by fixing the values of all polynomials on $\mathbf{t} / W$ whose pullback to $\mathbf{t}$ has degree $<h_{\mathfrak{g}}$. The induced $W$-cover of $\mathbb{P}_{w}^{1}$ then has $r=\operatorname{rank}(\mathfrak{g})$ branch points in $\mathbb{A}_{w}^{1}$, and one at $w=\infty$. The choice of a Borel labels the finite branch points $w_{\alpha}$ by the simple roots $\alpha$ in such a way that the monodromy at $w_{\alpha}$ is the reflection corresponding to $\alpha$. The monodromy at $\infty$ is then the product of these reflections, the Coxeter element. The Cameral cover is the pullback to $\mathbb{P}_{z}^{1}$. It can be described as the $W$-cover obtained from the trivial cover $W \times \mathbb{P}_{z}^{1}$ by making $r+1$ cuts labelled by the extended set of roots $\{\alpha\} \cup\left\{\alpha_{0}\right\}$, each running from a point $z_{\alpha}$ to $\mu z_{\alpha}^{-1}$, and pasting across each cut according to the corresponding element of $W$.

In addition to the loop algebras $\mathfrak{g}^{(1)}$, there are twisted versions $\mathfrak{g}^{(k)}$ where $k$ is the order of an automorphism of the Dynkin diagram of $\mathfrak{g}$, and each of these also gives rise to a periodic Toda system. The basic observation of Martinec and Warner [MW1] is that each of the known families of pure $N=2 \mathrm{SYM}$ curves arises as spectral curves for one of these twisted periodic Toda systems. The specific identification involves Langlands duality (Olive-Montonen duality, to a physicist) exchanging the direction of arrows in the extended diagram. The (untwisted) extended diagrams for types $A, D, E$ are self-dual, yielding ordinary Toda systems. The remaining cases yield twisted algebras and systems:

$$
\left(B_{r}^{(1)}\right)^{\vee}=A_{2 r-1}^{(2)},\left(C_{r}^{(1)}\right)^{\vee}=D_{r+1}^{(2)},\left(F_{4}^{(1)}\right)^{\vee}=E_{6}^{(2)},\left(G_{2}^{(1)}\right)^{\vee}=D_{4}^{(3)} .
$$

We will now describe explicitly the spectral curves for some of these systems.

\subsection{The spectral curves}

For the classical groups we can write the equation of the Toda spectral curves $C_{F, u}$ for the fundamental representation $F$ as

$$
w=z+\mu z^{-1}, x^{\epsilon} w+p=0
$$


where

$$
p=p(x)=x^{n+1}+u_{2} x^{n-1}+\cdots+u_{n+1}
$$

for type $A_{n}(G=\operatorname{SL}(n+1))$, and

$$
p=p\left(x^{2}\right)=x^{2 n}+u_{2} x^{2 n-2}+\ldots+u_{2 n}
$$

for types $B_{n}, C_{n}$, and $D_{n}$, and the $u_{\ell}$ are coordinates on the base. Here $\epsilon=0$ for $A_{n}, B_{n}, C_{n}$ and $\epsilon=2$ for $D_{n}$. In general, $\operatorname{deg}_{x}(p)$ is the dimension of the representation, while the $w$ term modifies the coefficient $u_{h_{\mathfrak{g}}}$ corresponding to the invariant of highest degree. For $A_{n}, B_{n}, C_{n}, D_{n}$, these Coxeter numbers are $n+1,2 n, 2 n, 2 n-2$. (The $B_{n}$ case is slightly different since 0 is a weight of $F$. So the actual "spectral" curve has equation $x(w+p(x))=0$. We may safely ignore the $x$ factor, since the distinguished Prym lives in the Jacobian of the other factor).

For $A_{n}$, the fundamental curve has genus $n$, so its Jacobian is the distinguished Prym. It can be described geometrically as the fiber product over $\mathbb{P}_{w}^{1}$ of the double cover $w=z+\mu z^{-1}$, branched at $w= \pm 2 \sqrt{\mu}$, and the degree $n+1$ cover $w=-p(x)$, with total ramification over $w=\infty$ and $n$ simple branch points at the images of the roots of $p^{\prime}(x)=0$. The substitution $y=2 z+p(x) / 2,4 \mu=\Lambda^{2(n+1)}$ converts it to

$$
y^{2}=p^{2}(x)-\Lambda^{2(n+1)},
$$

the form obtained in $[\mathrm{AF}]$ and [KLTY]. (Here we have reinstated the scale parameter $\Lambda$ of the asymptotically free theory. Previously this was set to 1 ).

The other spectral curves for $A_{n}$ are easily obtained from the fundamental one. The curve for $\wedge^{2} F$ is of degree $\frac{n(n+1)}{2}$ over the $z$ line, and of genus $n(n-1)+1-\left[\frac{n+1}{2}\right]$. The obvious correspondence between it and $C_{F, u}$ produces a copy of $J\left(C_{F, u}\right)$ in $J\left(C_{\wedge^{2} F, u}\right)$. The $A_{3}$ case is described in [Ml], [MS]: The tetragenal curve $C_{F, u}$ of genus 3 gives rise via Recillas' trigonal construction (cf. [D3]) to the degree 6 , genus 5 curve $C_{\wedge^{2} F, u}$, together with an involution whose quotient is a trigonal curve $C^{\prime}$ of genus 2. The Jacobian of $C_{F, u}$ is isogenous to $\operatorname{Prym}\left(C_{\wedge^{2} F, u} / C^{\prime}\right)$. (Actually, the degree 6 and 3 curves produced by the trigonal construction have genera 7 and 4 respectively, but each has 2 nodes, over $z=0$ and $z=\infty$; the curves $C_{\wedge^{2} F, u}$ and $C^{\prime}$, of genera 5 and 3 , are their respective desingularizations). A beautiful picture of the $g=11$ curve $C_{\wedge^{2} F, u}$ for $A_{4}$ is given in [MW1]. In contrast to these small curves, there is the gigantic cameral cover, of degree $n$ ! over $\mathbb{P}_{z}^{1}$ and of genus $1+\frac{n-1}{2(n+1)}(n+2)$ !, whose Jacobian contains those of all (irreducible components of) spectral covers.

For $D_{n}, C_{F, u}$ has equation $x^{2} w+p\left(x^{2}\right)=0$. This depends on $n$ parameters, but has genus $2 n-1$, too big. The quotient $C^{\prime}$ by the involution $x \mapsto-x$ is hyperelliptic of genus $n-1$; the $n$-dimensional piece is $\operatorname{Prym}\left(C_{F, u} / C^{\prime}\right)$, and it can be realized as the Jacobian of a curve $C^{\prime \prime}$, the quotient of $C_{F, u}$ by the involution $x \mapsto-x, z \mapsto \mu z^{-1}$. The substitution $y:=x^{2}\left(z-\mu z^{-1}\right), 4 \mu=$ $\Lambda^{4(n-1)}$ takes the equation of $C_{F, u}$ to

$$
y^{2}=p^{2}\left(x^{2}\right)-\Lambda^{4(n-1)} x^{4},
$$


the form of the $\mathrm{SO}(2 n) \mathrm{SYM}$ curve obtained in [BL]. In terms of $s=x y, t=x^{2}$, the quotient $C^{\prime \prime}$ becomes:

$$
s^{2}=t p^{2}(t)-\Lambda^{4(n-1)} t^{3} .
$$

For type $C_{n}$, the fundamental Toda curve $C_{F, u}$ has equation $w+p\left(x^{2}\right)=$ $0, w=z+\mu z^{-1}$. The substitution $y=2 z+p\left(x^{2}\right)$ changes this to

$$
y^{2}=p^{2}\left(x^{2}\right)-4 \mu \text {. }
$$

Just as in the $D_{n}$ case, $C_{F, u}$ has automorphism group $\mathbb{Z}_{2} \times \mathbb{Z}_{2}$ over the $t=x^{2}$ line:

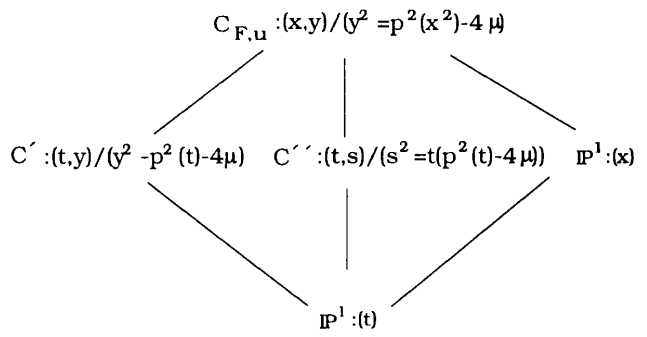

and the $n$-dimensional piece is $J\left(C^{\prime \prime}\right)$. But this is not the right abelian variety for the SYM theory. Rather, we should consider the Toda curves for $\left(C_{n}^{(1)}\right)^{\vee}=$ $D_{n+1}^{(2)}$. These have the form

$$
w^{2}+p\left(x^{2}\right)=0, w=z+\mu z^{-1} .
$$

The coordinates on the three $\mathbb{Z}_{2}$ quotients by the involutions $x \mapsto \pm x, z \mapsto$ $\pm \mu z^{-1}$ are now:

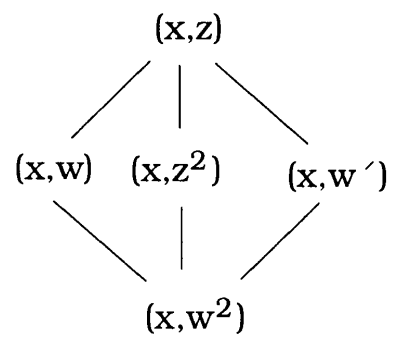

where $w^{\prime}=w-2 \mu z^{-1}=z-\mu z^{-1}$. The corresponding genera are:

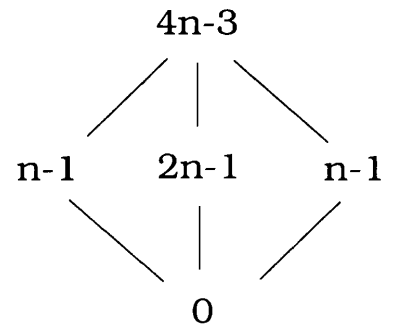


The middle curve has equation $v+\frac{\mu^{2}}{v}+p\left(x^{2}\right)-2 \mu=0$, where $v=z^{2}$. It factors further: in fact, it looks just like the $C_{n}$-Toda curve, with $z, \mu, p$ replaced by $v, \mu^{2}, p-2 \mu$ respectively. So its Jacobian has an $n$-dimensional piece, which is the Jacobian of:

$$
s^{2}=t\left((p(t)-2 \mu)^{2}-4 \mu^{2}\right)=t p(t)(p(t)-4 \mu) .
$$

This is essentially the $C_{n}$-curve obtained in [AS].

For $B_{n}$ the story is similar, but simpler. The dual Toda curve has equation $x\left(z+\mu z^{-1}\right)+p\left(x^{2}\right)=0$. The substitution $y=2 x z+p\left(x^{2}\right), 4 \mu=\Lambda^{2(2 n-1)}$ converts this to the form obtained in [DS],

$$
y^{2}=p^{2}\left(x^{2}\right)-\Lambda^{2(2 n-1)} x^{2} .
$$

Again this is of genus $2 n-1$. The genus $n$ quotient is

$$
s^{2}=t\left(p^{2}(t)-\Lambda^{2(2 n-1)} t\right) .
$$

For $G_{2}$, we need Toda curves for $\left(G_{2}^{(1)}\right)^{\vee}=D_{4}^{(3)}$. These are given [MW1] as:

$3\left(z-\mu z^{-1}\right)^{2}-x^{8}+2 u_{2} x^{6}-\left[u_{2}^{2}+6\left(z+\mu z^{-1}\right)\right] x^{4}+\left[u_{4}+2 u_{2}\left(z+\mu z^{-1}\right)\right] x^{2}=0$.

The various quotients involve the functions:

$$
\begin{aligned}
w & =z+\mu z^{-1} \\
s & =x^{2} \\
u & =x\left(z-\mu z^{-1}\right) \\
v & =x\left(w-s^{2}+\frac{u_{2}}{3} s\right) .
\end{aligned}
$$

Some quotient curves, indicated by the functions which generate their fields, are:

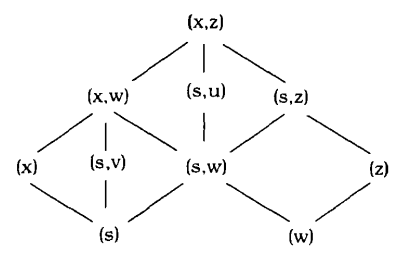

The corresponding genera are:

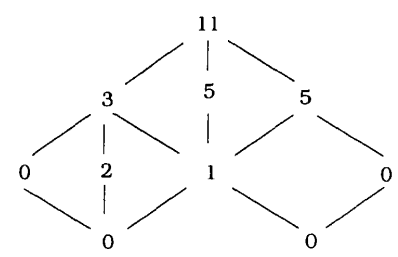


I have not checked whether the genus 2 curve with functions $(s, v)$ is the one corresponding to the $G_{2}$ theory. An explicit family of genus 2 curves was proposed in several works (see references in [AAG]) for the $G_{2}$ theory, but according to [LPG] this family does not have good physical properties, and does not match the Toda curves.

The $E_{6}$ curves were considered in [LW], and turn out to involve some beautiful geometry. The simplest curve is based on the 27-dimensional representation of $E_{6}$. The resulting 27-sheeted cover of the $z$ line behaves like the lines on a 1-parameter family of cubic surfaces. Each of the 6 simple roots corresponds to an ordinary double point acquired by the surface, so the local monodromy at each finite branch point is the product of 6 disjoint transpositions. Lerche and Warner study this genus-34 spectral curve, and go on to relate it to an integrable system coming from a string theory compactification on a Calabi-Yau threefold which degenerates to a fibration of the $E_{6}$ ALE singularity by a family of cubic surfaces.

\subsection{Fundamental matter}

The spectral curves for SYM with various gauge groups and numbers of particles ("quarks") in the fundamental representation have been determined by various means. A very incomplete list includes [HO], [APS], [AS], [H], [MN], [KP], [DKP1],[DKP2]. Each of these solutions is supported by substantial evidence, and yet the total picture seems far from complete. No really clear unifying principle is known, and there is no analogue of the Toda integrable system which is responsible for the various curves. There is also a certain level of disagreement among comparable solutions, see the discussion in [MN] and [DKP1]. (The curves involved in those solutions are the same, but the parametrizations differ. An integrable system would of course give a preferred parametrization).

\subsection{Adjoint matter}

As soon as $[\mathrm{MW}]$ and [DW] appeared, each with a class of integrable systems which solves its respective version of SYM, the question arose whether there was a common generalization: an integrable system for SYM with adjoint matter and arbitrary gauge group $G$, going to the periodic Toda system in the limit as $m$ and $\tau$ go together to $\infty$.

In [Mc], Martinec suggested that the elliptic Calogero-Moser system (cf. [OP]) may provide this common generalization. There is such a system for each semisimple $G$, and the case $G=\mathrm{SU}(n)$ agrees exactly with the system in [DW]. There is also a direct computation [I1] showing that the $A_{n}$ and $D_{n}$ systems can degenerate to the respective Toda systems, and there is a deformed family of integrable systems depending on some additional parameters (the elliptic spin models, [I2]). A way of obtaining the $A_{n}$ system by symplectic reduction is given in $[\mathrm{GN}]$.

One way to try to understand the geometry of these systems is as follows. 
Fix an elliptic curve $E$, a semisimple group $G$, and its maximal torus $T$. Let $\mathcal{M}, \mathcal{M}^{\prime}$ denote the moduli spaces of semistable bundles on $E$ with structue groups $G, T$ respectively. A special feature of genus 1 is that the natural map $\mathcal{M}^{\prime} \rightarrow \mathcal{M}$ is surjective, in fact it is a Galois cover with group $W$. (It is not true that the structure group of every semistable $G$-bundle on $E$ can be reduced to $T$; but every $S$-equivalence class of semistable bundles contains one whose structure group can be reduced). Now let $X$ be the moduli space of Higgs bundles on $E$ with first order pole at $\infty$, and let $X^{\prime}$ be its finite cover induced by $\mathcal{M}^{\prime} \rightarrow \mathcal{M}$. (A point of $X^{\prime}$ is an equivalence class of pairs $(V, \varphi)$ where $V$ is a $T$-bundle on $E$ and $\varphi$ is a meromorphic Higgs field on $\left.V \otimes_{T} G\right)$. There is a "moment map"

$$
\mu: X^{\prime} \rightarrow \mathbf{t}^{\perp} / T
$$

where $\mathbf{t}^{\perp} \subset \mathfrak{g}^{*}$ is the annihilator of the Cartan $\mathbf{t}$, and the $T$-action is coadjoint. Now all the fibers of $\mu$ are symplectic, of the minimal dimension $2 r$ (where $r=\operatorname{rank}(\mathfrak{g}))$, and at least for some of them (but certainly not for all!), the restrictions of all the $X$-Hamiltonians Poisson commute, giving a large family of small integrable systems. (Presumably, this involves an analogue of the AKS proof of integrability of the Toda system). As the elliptic curve degenerates to $\mathbb{P}^{1}$ with 0 glued to $\infty$, this construction specializes to the periodic Toda.

The question remains, though: of all the possible $T$-orbits, which one corresponds to SYM with adjoint matter? A necessary condition seems to be that the $T$ orbit $\mathcal{O}$ needs to be $W$-invariant; otherwise the limit as $m \rightarrow 0$ will not be the Hitchin system $T^{*} \mathcal{M}$, but its finite cover $T^{*} \mathcal{M}^{\prime}$. (Or something in-between, if $\mathcal{O}$ is invariant under a subgroup of $W$.) For $\mathrm{SU}(n)$, there is a unique such class (up to scalars), namely $\sum_{i \neq j} e_{i j}$. The natural analogue for arbitrary $\mathfrak{g}$ would be $\sum_{\alpha} e_{\alpha}$, the sum over all roots. Unfortunately, this is ill defined: the $e_{\alpha}$ can be normalized only up to sign, and different signs give distinct $T$ orbits. For the orthogonal groups, there does not seem to be any $W$-invariant $T$ orbit. David Lowe has pointed out [L] that for $S p(n)$ there is an invariant orbit, namely the $T$ orbit of the defining matrix $J$. (It is a signed sum of the long root vectors). This does give an integrable system, but a rather boring one: it is the $n$-fold product of the $\mathrm{SU}(2)$ system, which does not flow to the pure $N=2 C_{n}$ spectral curves described earlier.

In sum, the elliptic Calogero-Moser systems (or their spin variants) seem to be a promising general class of systems for realizing the SYM theory with adjoint matter for arbitrary gauge group, but at the moment we lack a good geometric understanding of these systems, e.g. via spectral curves, except in the case of $\mathrm{SU}(n)$.

\subsection{Stringy integrable systems?}

Quantum field theory is a limit of string theory compactified on a Calabi-Yau threefold, and the SW duality can be realized as the limiting form of various string dualities. A series of recent papers, such as [MW2], [KLMVW], [GHL1], 
[GHL2], [LW], suggest that much of the QFT structure studied in this paperthe spectral curves, integrable systems, SW differentials and so on-arises as limit of corresponding stringy structures. A possible framework for studying the transition from stringy integrable systems to the SW systems is proposed in $[F]$, where $S W$-type integrable systems are shown to arise from rigid special geometry, while the stringy type arises from local special geometry. From a purely mathematical point of view, it should be interesting to understand the relations between the SW-type systems, whose basic input is an elliptic curve; the Mukai system on a K3 surface, which degenerates to Hitchin's on a curve ([DEL], [DM1]); and the Calabi-Yau systems constructed in [DM2].

\section{Bibliography}

[AAG] M. R. Abolhasani, M. Alishahiha, A. M. Ghezelbash, hep-th 9606043 .

[AHH] M. Adams, J. Harnad, J. Hurtubise, Isospectral Hamiltonian flows in finite and infinite dimensions II: Integration of flows, Commun. Math. Phys. (1990), 555-585.

[AM] M. Adler, P. van Moerbeke, Completely integrable systems, Euclidean Lie algebras, and curves, Advances in Math. 38 (1980), 267-379.

[AF] P. C. Argyres, A. Faraggi, The vacuum Structure and Spectrum of $N=2$ Supersymmetric $S U(N)$ Gauge Theory, Phys. Rev. Lett. 74 (1995), hep-th 9411057.

[APS] P. C. Argyres, R. Plesser, A. D. Shapere, The Coulomb phase of $N=2$ supersymmetric $Q C D$, Phys. Rev. Lett. 75:1699-1702 (1995), no. e-Print Archive: hep-th 9505100.

[AS] P. C. Argyres, A. D. Shapere, The vacuum structure of $N=2$ super-QCD with classical gauge groups, hep-th 9509175 .

[B] A. Beauville, Jacobiennes des courbes spectrales et systemes hamiltoniens completement integrables, Acta Math. 164 (1990), 211-235.

[Be] J. Bernstein, Lectures on Supersymmetry, Lectures at IAS, Fall 1996, Preprint available at http://www.math.ias.edu/drm/QFT.

[Bo] F. Bottacin, Symplectic geometry on moduli spaces of stable pairs, Orsay, 1992.

[BL] A. Brandhuber, K. Landsteiner, On the Monodromies of $\mathrm{N}=2 \mathrm{Su}$ persymmetric Yang-Mills Theory with Gauge Group $S O(2 n)$, hepth 9507008 . 
[DS] U. H. Danielsson, B. Sundborg, The Moduli Space and Monodromies of $N=2$ Supersymmetric SO(2r+1) Yang-Mills Theory, hep-th 9504102 .

[DKP1] E. D'Hoker, I. M. Krichever, D. H. Phong, hep-th 9609041.

[DKP2] E. D'Hoker, I. M. Krichever, D. H. Phong, hep-th 9609145.

[D1] R. Donagi Decomposition of spectral covers, in Journees de Geometrie Algebrique D'Orsay, Asterisque 218 (1993), 145-175.

[D2] R. Donagi Spectral Covers Current Topics in Complex Algebraic Geometry, (H. Clemens and J. Kollar, eds.), MSRI Pub., vol. 28, 1995, pp. 65-86, Alg Geom, 9505009.

[D3] R. Donagi, The tetragonal construction Bull. Amer. Math. Soc. (N.S.) 4 (1981), 181-185.

[DEL] R. Donagi, L. Ein and R. Lazarsfeld, A non-linear deformation of the Hitchin dynamical system, AlgGeom 9504017.

[DM1] R. Donagi, E. Markman, Spectral curves, algebraically completely integrable Hamiltonian systems, and moduli of bundles, R. Donagi et al, Integrable systems and Quantum Groups, LNM 1620, (1996), 1-119 Alg Geom, 9507017.

[DM2] R. Donagi, E. Markman Cubics, Calabi-Yau threefolds, Integrable Systems and Mirrors, Israel Math. Conf. Proc., vol. 9, AMS, 1995, AlgGeom 9408004.

[DW] R. Donagi, E. Witten Supersymmetric Yang-Mills theory and integrable systems, Nucl. Phys B460 299 (1996), hep-th, 9510101.

[DH] J. J. Duistermaat, G. J. Heckman On the Variation in the Cohomology of the Symplectic Form of the Reduced Phase Space, Invent. Math. 69 (1982), 259.

[FS] L. D. Faddeev, A. A. Slavnov, Gauge Fields: Introduction to Quantum Theory, Benjamin/Cummings, 1980.

[Fa] G. Faltings, Stable G-bundles and Projective Connections, Jour. Alg. Geo 2 (1993), 507-568.

[F] D. S. Freed, On special Kähler manifolds, preprint.

[Fr] P. Freund, Introduction to supersymmetry, Camb. Monographs on Math. Phys., Camb. U. Press, 1986.

[GHL1] C. Gomez, R. Hernandez, E. Lopez, hep-th 9604057.

[GHL2] C. Gomez, R. Hernandez, E. Lopez, hep-th 9608104. 
[GKMMM] A. Gorsky, I. Krichever, A. Marshakov, A. Mironov, A. Morozov, hep-th 9505035.

[GN] A. Gorsky, N. Nekrasov, Elliptic Calogero-Moser system from twodimensional current algebra, hep-th 9401021.

[H] A. Hanany, On the quantum moduli space of vacua of $N=2$ supersymmetric gauge theories, hep-th 9509176.

[HO] A. Hanany, Y. Oz, On the quantum moduli space of vacua of $N=2$ supersymmetric $S U(N)$ gauge theories, hep-th 9505075.

[Hi] N. Hitchin, Stable bundles and integrable systems, Duke Math. J. 54 (1987), 91-114.

[I1] . I. Inozemtsev, The finite Toda lattices, Comm. Math. Phys. 121 (1989), 629-38.

[I2] V. I. Inozemtsev, Lax representation with spectral parameter on a torus for integrable particle systems, J. Lett. Math. Phys. 17 (1989), $11-17$.

[IM1] H. Itoyama, A. Morozov, hep-th 9511126.

[IM2] H. Itoyama, A. Morozov, hep-th 9512161.

[Ka] V. Kanev, Spectral curves, simple Lie algebras and Prym-Tjurin varieties, Proc. Symp. Pure Math. Part I 49 (1989), 627-645.

[KLMVW] A. Klemm, W. Lerche, P. Mayr, C. Vafa, N. Warner, hep-th 9604034 .

[KLTY] A. Klemm, W. Lerche, S. Theisen, S. Yankielowicz Simple Singularities and $N=2$ Supersymmetric Yang-Mills Theory Phys. Lett. 169 (1995), no. B344, hep-th 9411048.

[K] I. M. Krichever, hep-th 9205110.

[KP] I. M. Krichever, D. H. Phong, hep-th 9604199.

[LPG] K. Landsteiner, J. M. Pierre, S. B. Giddings, hep-th 9609059.

[LW] W. Lerche, N. P. Warner, hep-th 9608183.

[Mn] E. Markman, Spectral Curves and Integrable Systems, Comp. Math. 93 (1994), 255-290.

[Mv] A. Marshakov, hep-th 9607159.

[Mc] E. Martinec, hep-th 9510204. 
[MW1] E. Martinec, N. P. Warner Integrable Systems and Supersymmetric Gauge Theory, hep-th 9509161.

[MW2] E. Martinec, N. P. Warner, hep-th 9511052.

[Ml] A. McDaniel, Representations of $\operatorname{sl}(n, \mathbb{C})$ and the Toda lattice, Duke Math. J. 56 (1988), 47-99.

[MS] A. McDaniel, L. Smolinsky, A Lie theoretic Galois Theory for the spectral curves of an integrable system II, prep. (1994).

[Me] J. Y. Merindol, Varietes de Prym d'un Revetement Galoisien, prep. (1993).

[MN] J. A. Minahan, D. Nemeschansky, hep-th 9601059.

[MO] C. Montonen, D. Olive, Magnetic Monopoles as Gauge Particles?, Phys. Lett. 72B (1977), 177.

[Mu] S. Mukai, Symplectic structure of the moduli space of sheaves on an abelian or K3 surface, Inv. Math. 77 (1984), 101-116.

[NT] T. Nakatsu, K. Takasaki, hep-th 9509162.

[N] N. Nekrasov, Holomorphic bundles and many-body systems hep-th 9503157.

[OP] M. A. Olshanetski, A. M. Perelomov, Classical integrable finitedimensional systems related to Lie algebras, Phys. Rep. 71C (1981), 313-400.

[SW1] N. Seiberg, E. Witten, Monopole Condensation, and Confinement in $N=2$ Supersymmetric Yang-Mills Theory, Nucl. Phys. B426 (1994), 19, hep-th 9407087.

[SW2] N. Seiberg, E. Witten, Monopoles, duality and chiral symmetry breaking in $N=2$ supersymmetric $Q C D$, Nucl. Phys. B431 (1994), 484-550, hep-th 9408099.

[T] A. Treibich, Tangential polynomials and elliptic solitons, Duke Math. J. 59 (1989), 611-627.

[TV] A. Treibich, J. L. Verdier, Solitons Elliptiques, The Grothendieck Festschrift III (1990), Birkhäuser, 437-480.

[W] E. Witten, Monopoles and Four Manifolds, hep-th/9411102.

[WB] J. Wess, J. Bagger, Supersymmetry and supergravity, Princeton U. Press, 1992. 
